\title{
Association and Interaction Analysis of Body Mass Index and Triglycerides Level with Blood Pressure in Elderly Individuals in China
}

\author{
Lin Zhang, ${ }^{1}$ Jin-long Li, ${ }^{2}$ Li-li Zhang, ${ }^{3}$ Lei-lei Guo, ${ }^{1}$ Hong Li, ${ }^{1}$ and Dan Li ${ }^{4}$ \\ ${ }^{1}$ Department of Community Nursing, School of Nursing, Jinzhou Medical University, No. 40, Section 3, Songpo Road, \\ Linghe District Jinzhou City, Liaoning Province, China \\ ${ }^{2}$ Department of Occupational and Environmental Health, Key Laboratory of Occupational Health and Safety for Coal Industry in \\ Hebei Province, School of Public Health, North China University of Science and Technology, Tangshan, Hebei Province, China \\ ${ }^{3}$ Department of Surgery, Third Affiliated Hospital of Jinzhou Medical University, No. 28, Section 2, Chongqing Road, Linghe District, \\ Jinzhou City, Liaoning Province, China \\ ${ }^{4}$ Experimental Center for Nursing, School of Nursing, Jinzhou Medical University, No. 40, Section 3, Songpo Road, \\ Linghe District Jinzhou City, Liaoning Province, China
}

Correspondence should be addressed to Dan Li; 383869505@qq.com

Received 5 May 2018; Revised 11 September 2018; Accepted 4 October 2018; Published 22 November 2018

Academic Editor: Kazim Husain

Copyright (C) 2018 Lin Zhang et al. This is an open access article distributed under the Creative Commons Attribution License, which permits unrestricted use, distribution, and reproduction in any medium, provided the original work is properly cited.

Objectives. To assess the extent of interaction between body mass index (BMI) and triglyceride (TG) level and its effects on blood pressure (BP) in elderly individuals in China. Design. Cross-sectional study. Setting. Data were taken from a cross-sectional study called the China Health and Retirement Longitudinal Study. Participants. The analytic sample included 3629 subjects aged 45 to 96 years. Main Outcome Measurements. Data were obtained from the China Health and Retirement Longitudinal Study, which is a cross-sectional study. Age-adjusted partial Pearson's correlation test was used to compare various characteristics and BP. Adjusted associations were first used as linear regression models, as appropriate. Then, general linear models adjusted for related potential confounders were used to examine the synergistic effects of BMI and TG level on BP. Finally, a binary logistic regression model adjusted for confounding factors was used to examine the association between BMI or TG level and hypertension. Results. Ageadjusted partial Pearson's correlation coefficient showed that the TG level was positively correlated with both systolic blood pressure (SBP) and diastolic blood pressure (DBP) in both men and women with BMI $<24.0 \mathrm{~kg} / \mathrm{m}^{2}$; however, TG level was positively correlated with DBP in women with $\mathrm{BMI} \geq 24.0 \mathrm{~kg} / \mathrm{m}^{2}$ but not with DBP in men with BMI $\geq 24.0 \mathrm{~kg} / \mathrm{m}^{2}$. Multiple linear regression analysis showed that BMI level was significantly and positively associated with both SBP and DBP in men and women with BMI $<24.0 \mathrm{~kg} / \mathrm{m}^{2}$, and TG level was significantly and positively associated with SBP in women with BMI $<24.0 \mathrm{~kg} / \mathrm{m}^{2}$, independent of other confounding factors. A general linear model analysis with adjustment for confounding factors (age, educational level, marital status, current residence, smoking, eating habits, taking activities, antidiabetic medication, antihypertensive therapy, fasting plasma glucose [FPG], low-density lipoprotein cholesterol [LDL-C], estimated glomerular filtration rate [eGFR], and serum uric acid [SUA]) showed no interaction between BMI and TG level and SBP (men, $\beta=0.572, P=0.845$; women, $\beta=0.122, P=$ 0.923 ) and DBP (men, $\beta=-0.373, P=0.810$; women, $\beta=0.272, P=0.828$ ). A binary logistic regression model analysis with adjustment for confounding factors (age, educational level, marital status, current residence, smoking, drinking, eating habits, taking activities, major accidental injury, physical activity, history of cardiovascular disease, history of liver disease, antilipidemic medication, antidiabetic medication, antihypertensive therapy, FPG, LDL-C, high-density lipoprotein cholesterol [HDL-C], eGFR, and SUA) showed that overweight and obese men and women were more likely to have hypertension (men: odds ratio [OR] = $1.781,95 \%$ confidence interval $[\mathrm{CI}]=1.393-2.277$; women: $\mathrm{OR}=1.653,95 \% \mathrm{CI}=1.330-2.055$ ) and women with high TG were more likely to have hypertension $(\mathrm{OR}=1.558,95 \% \mathrm{CI}=1.219-1.992)$. Conclusion. An interactive effect of BMI and TG level on BP was not observed in either men or women; however, independent effects of BMI on BP were observed in both men and women, and an association between TG level and hypertension was observed in women. 


\section{Introduction}

The prevalence of hypertension has dramatically increased in the past few years in China[1,2]; furthermore, the rate of hypertension is disproportionately high among elderly individuals in this country[3-5]. Hypertension is defined as a diastolic blood pressure (DBP) of $\geq 90 \mathrm{mmHg}$ and/or systolic blood pressure (SBP) of $\geq 140 \mathrm{mmHg}$ based on the evidence of modestly increasing risk[6-8] and incidence of cardiovascular disease (CVD) [9-11].

Hypertension is a complex disease, and patients with the condition suffer from an economic, psychosocial, and physical burden. Recently, hypertension has become an important global public health challenge [12]. Therefore, an effective strategy to prevent hypertension and determine its associated risks should be carefully implemented. Numerous studies [1320] have been conducted to determine the risk factors for hypertension, such as aging, overweight, central obesity, lifestyle, family history of hypertension, history of diabetes and dyslipidemia, lack of physical activity, smoking, alcohol consumption, low-density lipoprotein cholesterol (LDL-C) level, triglycerides (TG) level, and high-density lipoprotein cholesterol (HDL-C) level. Lipid abnormalities have been shown to increase the risk factors for hypertension [21]. For example, Teng [22] found an additive effect of TG on DBP. Brennan [23] conducted a study to investigate the effects of body weight on TG and SBP in individuals aged 20 to 49 years and found that, in men, significant correlations with BMI were found for all variables, while in women younger than 40 , only the correlation with BP was significant. TG level has been associated with BP since TG level itself can lead to endothelial dysfunction [24, 25], arterial stiffness [26], and loss of vasomotor reactivity [27]. However, it is also important to understand the interrelationships among various risk factors for hypertension. In particular, the association between TG level and risk of hypertension and the effects of obesity on this association are of considerable interest, and an interactive effect between body mass index (BMI) and TG level on BP may also be considered.

No consistent recognition of the association and interaction analysis between BMI and TG level and BP in elderly individuals exists. Thus, the present study particularly aimed to determine the prevalence of normotension and hypertension and their association with BMI, TG level, and other confounding factors based on sex, using cross-sectional data from community-dwelling individuals aged $\geq 60$ years in China.

\section{Methods}

2.1. Study Design and Setting. Data for this cross-sectional study were taken from the China Health and Retirement Longitudinal Study (CHARLS), a biennial and nationally representative longitudinal survey conducted by the China Centre for Economic Research at Peking University [28]. The baseline survey had a four-stage, stratified, cluster probability sampling design.
In the first stage, all counties in China were stratified by region, rural/urban status, and gross domestic product per capita. A random sample of 150 counties was selected to represent the socioeconomic and geographic pattern of all the counties. In the second stage, three primary sampling units (PSUs) were selected in each county with the probability of inclusion of each county in the sample proportional to their population size. In the third stage, all households in each selected PSU were mapped, and a random sample of 24 households was selected among all the households with residents aged $\geq 45$ years within each PSU. Finally, for each selected household, one resident aged $\geq 45$ years was randomly selected as a participant in the survey. From the 2011 CHARLS Wavel, we included a total of 3629 individuals in our study.

2.2. Information on Demographic Characteristics and SelfReported Risk Factors. Data including age, education, marital status, current residence, smoking, drinking, eating habits, taking activities, accidental injury, physical exercise, history of cardiovascular disease, history of liver disease, antilipidemic medication, antidiabetic medication, and antihypertensive therapy were obtained using a self-reported questionnaire. (1) Median age was 68 years, and age was categorized as $<68$ years and $\geq 68$ years. (2) Educational levels were classified into illiterate, less than elementary school, high school, and above vocational school. (3) Marital status was classified into married and single. (4) Current residence was classified into rural and urban. (5) Smoking status was never smoked, ex-smoker, and current smoker. (6) Alcohol consumption was classified into more than non-drinker, less than once a month, and once a month. (7) Eating habits were categorized into 2 meals per day or fewer, 3 meals per day, and 4 meals per day or more. (8) Activity status was dichotomized into at least once a month versus never. (9) Major accidental injury information was obtained by asking the participant whether he/she suffered from any type of major accidental injury and received medical treatment; the answer was "yes" or "no." (10) Regular physical exercise was defined as exercising at least 3 days per week and more than 30 minutes per day, including moderate to vigorous physical activity and walking. (11) History of CVD, history of liver disease, antilipidemic medication, antidiabetic medication, and antihypertensive therapy were defined as a history of receiving treatment for the respective diseases; the answer was "yes" or "no."

2.3. Glucose, LDL, HDL, Triglycerides, eGFR, BP, and Uric Acid Measurement. Venous blood samples were obtained at the Centers for Disease Control and Prevention (CDC) station, then immediately stored and frozen at $-20^{\circ} \mathrm{C}$, and transported within 2 weeks to the Chinese CDC in Beijing, where they were placed in a deep freezer and stored at $-80^{\circ} \mathrm{C}$ until the relevant assay was performed at the China Medical University laboratory. (1) Fasting plasma glucose (FPG), LDL, HDL, and TG levels were analyzed at the Youanmen Center for Clinical Laboratory at Capital Medical University using the enzymatic colorimetric tests, and serum uric acid 
(SUA) levels were analyzed using the urinalysis (UA) plus method. We classified TG levels into 2 categories: $\geq 150$ $\mathrm{mg} / \mathrm{dL}$ and $<150 \mathrm{mg} / \mathrm{dL}$, a categorization widely used in previous studies $[29,30]$. (2) BP was measured 3 times at intervals of 45 seconds with a sphygmomanometer. The value of BP was determined based on the mean of the 3 measurements. Normotension was defined as absence of antihypertensive therapy with an SBP of $<140 \mathrm{mmHg}$ and DBP of $<90 \mathrm{mmHg}$, while hypertension was defined as an SBP of $\geq 140 \mathrm{mmHg}$ and/or DBP of $\geq 90 \mathrm{mmHg}$; this categorization has been widely used in previous studies. (3) Estimated glomerular filtration rate (eGFR) was calculated using the CKD-EPI creatinine-cystatin equations [31]: (1) in men, serum creatinine $(\mathrm{Scr}) \leq 0.9$, serum cystatin $\mathrm{C}(\mathrm{Scys}) \leq 0.8$, eGFR $=135 *(\mathrm{Scr} / 0.9)^{-0.207} *$ $(\text { Scys } / 0.8)^{-0.375} * 0.995^{\text {age }} ; \mathrm{Scr} \leq 0.9$, Scys $>0.8$, eGFR $=135 *$ $(\mathrm{Scr} / 0.9)^{-0.207} *(\mathrm{Scys} / 0.8)^{-0.711} * 0.995^{\text {age }} ; \mathrm{Scr}>0.9, \mathrm{Scys} \leq 0.8$, $\mathrm{eGFR}=135 * \quad(\mathrm{Scr} / 0.9)^{-0.601} * \quad(\mathrm{Scys} / 0.8)^{-0.375} * 0.995^{\text {age }}$; Scr $>0.9$, Scys $>0.8$, eGFR $=135 *(\mathrm{Scr} / 0.9)^{-0.601} *$ $(\text { Scys } / 0.8)^{-0.711} * 0.995^{\text {age }} ;$ (2) in women, Scr $\leq 0.7$, Scys $\leq 0.8, \mathrm{eGFR}=130 *(\mathrm{Scr} / 0.7)^{-0.248} *(\mathrm{Scys} / 0.8)^{-0.375} * 0.995^{\text {age }}$; Scr $\leq \quad 0.7, \quad$ Scys $>0.8$, and eGFR = $130 *(\mathrm{Scr} / 0.7)^{-0.248} *(\mathrm{Scys} / 0.8)^{-0.711} * 0.995^{\text {age }} ; \mathrm{Scr}>0.7$, Scys $\leq$ 0.8, eGFR $=130 *(\mathrm{Scr} / 0.7)^{-0.601} *(\mathrm{Scys} / 0.8)^{-0.375} * 0.995^{\text {age }}$; Scr $>0.7$, Scys $>0.8$, and eGFR $=130 *(\text { Scr } / 0.7)^{-0.601} *$ (Scys $/ 0.8)^{-0.711} * 0.995^{\text {age }}$.

2.4. Measurement of Body Mass Index. Weight and height were measured using a weight and height measurement instrument. BMI was calculated based on the measured weight and height of the participants, who were classified into four categories: underweight (BMI, $\leq 18.5 \mathrm{~kg} / \mathrm{m}^{2}$ ), normal weight $\left(18.5-24 \mathrm{~kg} / \mathrm{m}^{2}\right)$, overweight $\left(24-28 \mathrm{~kg} / \mathrm{m}^{2}\right)$, and obese $\left(>28 \mathrm{~kg} / \mathrm{m}^{2}\right)$ [32].

2.5. Statistical Analysis. Our data are represented as mean \pm standard deviation (SD; continuous data) and number and percentage (categorical data). Differences between normotension and hypertension, or between normal weight individuals and those with underweight or adiposity, were evaluated using the t-test or chi-square test, followed by Bonferroni adjustment. Correlations between various characteristics and BP were compared using the age-adjusted partial Pearson's correlation test. The adjusted associations between various characteristics and BP were first compared using linear regression models, as appropriate. Then, general linear models adjusted for related potential confounders (age, educational level, marital status, current residence, smoking, drinking, eating habits, taking activities, major accidental injury, physical activity, history of CVD, history of liver disease, antilipidemic medication, antidiabetic medication, antihypertensive therapy, FPG, LDL-C, HDL-C, eGFR, and SUA) [33] were constructed to examine the synergistic effect of BMI and TG level on BP. A binary logistic regression model adjusted for related potential confounders was used to examine the association between BMI or TG and hypertension. A $P$-value of 0.05 was considered significant. All data were analyzed using the SPSS version 17.0 (IBM Corp., Armonk, NY, USA).

2.6. Patient and Public Involvement Statement. Consent from the respondents was obtained by the CHARLS. The data information obtained from the study was public, and patients were not involved.

\section{Results}

The 2011 CHARLS Wavel sample ( $\mathrm{N}=3629)$ consists of $49.88 \%$ men $($ Age: Mean $=68.86$ years; $\mathrm{SD}=6.30$; range, $60-93$ years) and $50.12 \%$ women (Age: Mean $=68.55$ years; $\mathrm{SD}=$ 6.82; range, 60-96 years). Among men, the mean SBP and DBP were $128.93 \mathrm{mmHg}$ and $72.28 \mathrm{mmHg}$, respectively, and among women, the mean SBP and DBP were $133.66 \mathrm{mmHg}$ and $73.00 \mathrm{mmHg}$, respectively. In men, $10.94 \%, 60.66 \%$, $21.60 \%$, and $6.80 \%$ were underweight, normal weight, overweight, and obese, respectively, whereas in women $10.34 \%$, $49.04 \%, 29.08 \%$, and $11.54 \%$ were underweight, normal weight, overweight, and obese, respectively. The mean and SD of TG level were $112.79 \pm 83.55 \mathrm{mg} / \mathrm{dL}$ in men and $140.14 \pm 94.12 \mathrm{mg} / \mathrm{dL}$ in women. Tables 1 and 2 present the baseline characteristics of the sample for all variables, and most variables were based on our previous research [32].

Tables 1 and 2 show the various characteristics of participants categorized on basis of BMI. The participants comprised 1,810 men aged $68.86 \pm 6.30$ (range: 60-93) years and 1,819 women aged $68.55 \pm 6.82$ (range, 60-96) years. According to the modified Chinese criteria for BMI [34], the mean BMI in men was $22.41 \mathrm{~kg} / \mathrm{m}^{2}$ (SD, 3.71), with $10.94 \%$ underweight $\left(\mathrm{BMI},<18.5 \mathrm{~kg} / \mathrm{m}^{2}\right), 60.66 \%$ normal weight (BMI, $18.5-24 \mathrm{~kg} / \mathrm{m}^{2}$ ), $21.60 \%$ overweight, and $6.80 \%$ obese (BMI, $\geq 28 \mathrm{~kg} / \mathrm{m}^{2}$ ), whereas the mean BMI in women was $23.33 \mathrm{~kg} / \mathrm{m}^{2}$ (SD, 4.23), with 10.34\% underweight (BMI, $<18.5$ $\mathrm{kg} / \mathrm{m}^{2}$ ), $49.04 \%$ normal weight (BMI, $18.5-24 \mathrm{~kg} / \mathrm{m}^{2}$ ), $29.08 \%$ overweight, and $11.54 \%$ obese (BMI, $\geq 28 \mathrm{~kg} / \mathrm{m}^{2}$ ). Table 1 shows the background characteristics of male participants categorized based on BMI. Levels of FPG, LDL-C, TG, eGFR, SUA, SBP, and DBP were significantly higher in the high BMI group than in the low BMI group, whereas HDL$C$ level was higher in the low BMI group than in the high BMI group. However, between-group differences in the prevalence of major accidental injury, regular physical exercise, history of liver disease, and antihypertensive therapy were not observed. In women, levels of FBG, LDL-C, TG, eGFR, SUA, SBP, and DBP were significantly higher in the high BMI group than those in the low BMI group, but HDL-C level was higher in the low BMI group than in the high BMI group. However, between-group differences in categories of alcohol drinking, major accidental injury, physical activity, and history of liver disease were not observed (Table 2).

Tables 3 and 4 show the characteristics of participants categorized by BMI and BP status. First, in the hypertensive group with a $\mathrm{BMI}<24.0 \mathrm{~kg} / \mathrm{m}^{2}$ in men, levels of glucose, LDL-C, BMI, SUA, SBP, and DBP were significantly higher than those in the hypertensive group, but eGFR level was significantly higher than that in the normotensive group. 
TABLE 1: Various characteristics of participants categorized by BMI in male $(\mathrm{N}=1810)$.

\begin{tabular}{|c|c|c|c|c|c|c|}
\hline Variables & $\begin{array}{c}\mathrm{BMI} \leq 18.5 \\
(\mathrm{n}=198)\end{array}$ & BMI18.5-24 $(\mathrm{n}=1098)$ & BMI 24-28 (n=391) & $\begin{array}{l}\mathrm{BMI}>28 \\
(\mathrm{n}=123)\end{array}$ & $t / \chi^{2}$ & $P$ \\
\hline Age(years) & $71.49 \pm 6.31$ & $69.17 \pm 6.4$ & $67.35 \pm 5.71$ & $66.7 \pm 5.22$ & 25.817 & $<0.001$ \\
\hline \multicolumn{7}{|l|}{ Education } \\
\hline Illiterate & $52(26.26)$ & $256(23.32)$ & $44(11.25)$ & $20(16.26)$ & \multirow{4}{*}{42.976} & \multirow{4}{*}{$<0.001$} \\
\hline Less than elementary school & $130(65.66)$ & $773(70.40)$ & $306(78.26)$ & $90(73.17)$ & & \\
\hline High school & $6(3.03)$ & $17(1.55)$ & $5(1.28)$ & $4(3.25)$ & & \\
\hline Above vocational school & $10(5.05)$ & $52(4.74)$ & $36(9.21)$ & $9(7.32)$ & & \\
\hline \multicolumn{7}{|l|}{ Marital status } \\
\hline Single & $29(14.65)$ & $179(16.30)$ & $30(7.67)$ & $13(10.57)$ & \multirow[t]{2}{*}{19.2333} & \multirow[t]{2}{*}{$<0.001$} \\
\hline Married & $169(85.35)$ & $919(83.70)$ & $361(92.33)$ & $110(89.43)$ & & \\
\hline \multicolumn{7}{|l|}{ Current residence } \\
\hline Rural & $152(76.77)$ & 791(72.04) & $213(54.48)$ & $65(52.85)$ & \multirow[t]{2}{*}{60.303} & \multirow[t]{2}{*}{$<0.001$} \\
\hline Urban & $46(23.23)$ & $307(27.96)$ & $178(45.52)$ & $58(47.15)$ & & \\
\hline \multicolumn{7}{|l|}{ Smoke } \\
\hline $\mathrm{NO}$ & $118(59.60)$ & $632(57.56)$ & $163(41.69)$ & $48(39.02)$ & \multirow[t]{3}{*}{43.161} & \multirow[t]{3}{*}{$<0.001$} \\
\hline Former smoke & $36(18.18)$ & 199(18.12) & $105(26.85)$ & $33(26.83)$ & & \\
\hline Current smoke & $44(22.22)$ & $267(24.32)$ & $123(31.46)$ & $42(34.15)$ & & \\
\hline \multicolumn{7}{|l|}{ Drinking } \\
\hline $\mathrm{NO}$ & $115(58.08)$ & $543(49.45)$ & $202(51.66)$ & $65(52.85)$ & \multirow[t]{3}{*}{197.72} & \multirow[t]{3}{*}{$<0.001$} \\
\hline Less than once a month & $13(6.57)$ & $94(8.56)$ & $33(8.44)$ & 14(11.38) & & \\
\hline More than once a month & $70(35.35)$ & $461(41.99)$ & $156(39.9)$ & $44(35.77)$ & & \\
\hline \multicolumn{7}{|l|}{ Eating meals } \\
\hline$\leq 2$ meals per day & $31(15.66)$ & $174(15.85)$ & $43(11.00)$ & $10(8.13)$ & 15.580 & 0.016 \\
\hline 3 meals per day & $162(81.82)$ & $905(82.42)$ & $345(88.24)$ & $113(91.87)$ & & \\
\hline$\geq 4$ meals per day & $5(2.53)$ & 19(1.73) & $3(0.77)$ & $0(0.00)$ & & \\
\hline Taking activities & & & & & & \\
\hline No & $119(60.10)$ & $593(54.01)$ & $175(44.76)$ & $62(50.41)$ & 15.198 & 0.002 \\
\hline Yes & $79(39.90)$ & $505(45.99)$ & $216(55.24)$ & $61(49.59)$ & & \\
\hline Ever been in major accidental injury & & & & & & \\
\hline No & $173(87.37)$ & 976(88.89) & $349(89.26)$ & $111(90.24)$ & 0.743 & 0.863 \\
\hline Yes & $25(12.63)$ & $122(11.11)$ & $42(10.74)$ & $12(9.76)$ & & \\
\hline Having regular physical exercises & & & & & & \\
\hline No physical exercise & $122(61.62)$ & $701(63.84)$ & $247(63.17)$ & $69(56.1)$ & 5.204 & 0.518 \\
\hline Less than regular physical exercises & $39(19.70)$ & $183(16.67)$ & $60(15.35)$ & $23(18.70)$ & & \\
\hline Regular physical exercises & $37(18.69)$ & 214(19.49) & $84(21.48)$ & $31(25.20)$ & & \\
\hline History of CVD & & & & & & \\
\hline No & $178(89.90)$ & $965(87.89)$ & $323(82.61)$ & $87(70.73)$ & 33.007 & $<0.001$ \\
\hline Yes & $20(10.10)$ & 133(12.11) & $68(17.39)$ & $36(29.27)$ & & \\
\hline History of liver diseases & & & & & & \\
\hline No & 194(97.98) & $1055(96.08)$ & $375(95.91)$ & $114(92.68)$ & 5.576 & 0.134 \\
\hline Yes & $4(2.02)$ & $43(3.92)$ & $16(4.09)$ & $9(7.32)$ & & \\
\hline Antilipidemic medication & & & & & & \\
\hline No & 194(97.98) & 1063(96.81) & $366(93.61)$ & $104(84.55)$ & 43.969 & $<0.001$ \\
\hline Yes & $4(2.02)$ & $35(3.19)$ & $25(6.39)$ & $19(15.45)$ & & \\
\hline Anti-diabetic medication & & & & & & \\
\hline No & 195(98.48) & $1069(97.36)$ & $365(93.35)$ & $104(84.55)$ & 54.001 & $<0.001$ \\
\hline Yes & $3(1.52)$ & $29(2.64)$ & $26(6.65)$ & $19(15.45)$ & & \\
\hline Anti-hypertensive therapy & & & & & & \\
\hline No & $186(93.94)$ & 1036(94.35) & $359(91.82)$ & $114(92.68)$ & 3.345 & 0.341 \\
\hline Yes & $12(6.06)$ & $62(5.65)$ & $32(8.18)$ & $9(7.32)$ & & \\
\hline Fasting plasma glucose(mg/dl) & $105.00 \pm 28.94$ & $109.16 \pm 36.00$ & $118.37 \pm 41.50$ & $119.64 \pm 41.26$ & 9.994 & $<0.001$ \\
\hline LDL Cholesterol (mg/dl) & $105.37 \pm 32.12$ & $110.54 \pm 32.35$ & $118.02 \pm 35.30$ & $119.42 \pm 34.05$ & 9.701 & $<0.001$ \\
\hline HDL Cholesterol (mg/dl) & $60.04 \pm 16.14$ & $53.83 \pm 15.90$ & $44.00 \pm 13.320$ & $42.9 \pm 12.28$ & 74.457 & $<0.001$ \\
\hline $\mathrm{eGFR}\left(\mathrm{ml} / \mathrm{min} / 1.73 \mathrm{~m}^{2}\right)$ & $71.56 \pm 16.22$ & $74.92 \pm 16.53$ & $75.57 \pm 16.32$ & $75.13 \pm 16.23$ & 2.868 & 0.035 \\
\hline Serum uric acid(mg/dl) & $4.79 \pm 1.29$ & $4.98 \pm 1.31$ & $5.37 \pm 1.34$ & $5.27 \pm 1.29$ & 12.990 & $<0.001$ \\
\hline Systolic blood pressure (mmHg) & $128.93 \pm 21.56$ & $132.59 \pm 24.29$ & $139.25 \pm 25.58$ & $143.94 \pm 19.85$ & 23.845 & $<0.001$ \\
\hline Diastolic blood pressure $(\mathrm{mmHg})$ & $72.28 \pm 12.34$ & $74.59 \pm 12.78$ & $78.65 \pm 12.37$ & $81.69 \pm 12.29$ & 17.079 & $<0.001$ \\
\hline Body mass index $\left(\mathrm{kg} / \mathrm{m}^{2}\right)$ & $17.24 \pm 0.90$ & $21.25 \pm 1.53$ & $25.65 \pm 1.12$ & $30.71 \pm 4.3 .00$ & 2126.849 & $<0.001$ \\
\hline Triglycerides (mg/dl) & $84.34 \pm 34.71$ & $101.52 \pm 59.33$ & $146.98 \pm 129.67$ & $150.5 \pm 92.37$ & 47.962 & $<0.001$ \\
\hline
\end{tabular}


TABLE 2: Various characteristics of participants categorized by BMI in female $(\mathrm{N}=1819)$.

\begin{tabular}{|c|c|c|c|c|c|c|}
\hline Variables & $\begin{array}{c}\mathrm{BMI} \leq 18.5 \\
(\mathrm{n}=188)\end{array}$ & BMI18.5-24 (n=892) & BMI 24-28 $(\mathrm{n}=529)$ & $\begin{array}{l}\text { BMI }>28 \\
(n=210)\end{array}$ & $t / \chi^{2}$ & $P$ \\
\hline Age (years) & $71.41 \pm 7.07$ & $68.77 \pm 6.99$ & $67.64 \pm 6.32$ & $67.34 \pm 6.25$ & 17.169 & $<0.001$ \\
\hline \multicolumn{7}{|l|}{ Education } \\
\hline Illiterate & $133(70.74)$ & $530(59.42)$ & $269(50.85)$ & $111(52.86)$ & \multirow{4}{*}{35.807} & \multirow{4}{*}{$<0.001$} \\
\hline Less than elementary school & $55(29.26)$ & $342(38.34)$ & $236(44.61)$ & $97(46.19)$ & & \\
\hline High school & $0(0.00)$ & $6(0.67)$ & $9(1.70)$ & $0(0.00)$ & & \\
\hline Above vocational school & $0(0.00)$ & $14(1.57)$ & $15(2.84)$ & $2(0.95)$ & & \\
\hline \multicolumn{7}{|l|}{ Marital status } \\
\hline Single & $72(38.30)$ & $291(32.62)$ & $131(24.76)$ & $49(23.33)$ & \multirow[t]{2}{*}{20.478} & \multirow[t]{2}{*}{$<0.001$} \\
\hline Married & $116(61.70)$ & $601(67.38)$ & $398(75.24)$ & $161(76.67)$ & & \\
\hline \multicolumn{7}{|l|}{ Current residence } \\
\hline Rural & 150(79.79) & $588(65.92)$ & $309(58.41)$ & $107(50.95)$ & \multirow[t]{2}{*}{43.911} & \multirow[t]{2}{*}{$<0.001$} \\
\hline Urban & $38(20.21)$ & $304(34.08)$ & $220(41.59)$ & 103(49.05) & & \\
\hline \multicolumn{7}{|l|}{ Smoke } \\
\hline NO & $151(80.32)$ & $783(87.78)$ & $479(90.55)$ & $190(90.48)$ & \multirow[t]{3}{*}{20.377} & \multirow[t]{3}{*}{$<0.001$} \\
\hline Former smoke & $6(3.19)$ & $30(3.36)$ & $15(2.84)$ & $8(3.81)$ & & \\
\hline Current smoke & $31(16.49)$ & $79(8.86)$ & $35(6.62)$ & $12(5.71)$ & & \\
\hline \multicolumn{7}{|l|}{ Drinking } \\
\hline NO & $164(87.23)$ & $781(87.56)$ & $462(87.33)$ & 194(92.38) & \multirow[t]{3}{*}{7.632} & \multirow[t]{3}{*}{0.266} \\
\hline Less than once a month & $10(5.32)$ & $32(3.59)$ & $27(5.10)$ & $6(2.86)$ & & \\
\hline More than once a month & $14(7.45)$ & $79(8.86)$ & $40(7.56)$ & $10(4.76)$ & & \\
\hline \multicolumn{7}{|l|}{ Eating meals } \\
\hline$\leq 2$ meals per day & $48(25.53)$ & $143(16.03)$ & $58(10.96)$ & $18(8.57)$ & 32.785 & $<0.001$ \\
\hline 3 meals per day & $139(73.94)$ & $732(82.06)$ & $463(87.52)$ & $189(90.00)$ & & \\
\hline$\geq 4$ meals per day & $1(0.53)$ & $17(1.91)$ & $8(1.51)$ & $3(1.43)$ & & \\
\hline Taking activities & & & & & & \\
\hline No & $113(60.11)$ & $490(54.93)$ & $241(45.56)$ & $100(47.62)$ & 19.191 & $<0.001$ \\
\hline Yes & $75(39.89)$ & $402(45.07)$ & $288(54.44)$ & $110(52.38)$ & & \\
\hline Ever been in major accidental injury & & & & & & \\
\hline No & $173(92.02)$ & $830(93.05)$ & $490(92.63)$ & 197(93.81) & 0.573 & 0.903 \\
\hline Yes & 15(7.98) & $62(6.95)$ & $39(7.37)$ & $13(6.19)$ & & \\
\hline Having regular physical exercises & & & & & & \\
\hline No physical exercise & $134(71.28)$ & $569(63.79)$ & $322(60.87)$ & $132(62.86)$ & 9.032 & 0.172 \\
\hline Less than regular physical exercises & $25(13.30)$ & $172(19.28)$ & $99(18.71)$ & $36(17.14)$ & & \\
\hline Regular physical exercises & $29(15.43)$ & $151(16.93)$ & $108(20.42)$ & $42(20.00)$ & & \\
\hline History of CVD & & & & & & \\
\hline No & $160(85.11)$ & $757(84.87)$ & $428(80.91)$ & $158(75.24)$ & 13.931 & 0.003 \\
\hline Yes & $28(14.89)$ & $135(15.13)$ & 101(19.09) & $52(24.76)$ & & \\
\hline History of liver diseases & & & & & & \\
\hline No & 183(97.34) & $864(96.86)$ & $504(95.27)$ & 203(96.67) & 3.289 & 0.349 \\
\hline Yes & $5(2.66)$ & $28(3.14)$ & $25(4.73)$ & $7(3.33)$ & & \\
\hline Antilipidemic medication & & & & & & \\
\hline No & $185(98.40)$ & $854(95.74)$ & $474(89.60)$ & $178(84.76)$ & 49.744 & $<0.001$ \\
\hline Yes & $3(1.60)$ & $38(4.26)$ & $55(10.40)$ & $32(15.24)$ & & \\
\hline Anti-diabetic medication & & & & & & \\
\hline No & 187(99.47) & $863(96.75)$ & $490(92.63)$ & $188(89.52)$ & 33.154 & $<0.001$ \\
\hline Yes & $1(0.53)$ & $29(3.25)$ & $39(7.37)$ & $22(10.48)$ & & \\
\hline Anti-hypertensive therapy & & & & & & \\
\hline No & $171(90.96)$ & $838(93.95)$ & 495(93.57) & 191(90.95) & 33.154 & $<0.001$ \\
\hline Yes & $17(9.04)$ & $54(6.05)$ & $34(6.43)$ & $19(9.05)$ & & \\
\hline Fasting plasma glucose (mg/dl) & $105.18 \pm 22.29$ & $110.63 \pm 40.95$ & $118.34 \pm 48.98$ & $116.55 \pm 35.01$ & 6.659 & $<0.001$ \\
\hline LDL Cholesterol (mg/dl) & $115.41 \pm 33.67$ & $122.84 \pm 34.65$ & $127.66 \pm 38.90$ & $128.45 \pm 41.61$ & 6.511 & $<0.001$ \\
\hline HDL Cholesterol (mg/dl) & $60.31 \pm 16.04$ & $54.55 \pm 15.13$ & $47.67 \pm 13.02$ & $45.26 \pm 11.70$ & 62.326 & $<0.001$ \\
\hline eGFR (ml/min/1.73m²) & $72.49 \pm 16.54$ & $75.97 \pm 16.10$ & $77.40 \pm 15.8$ & $75.16 \pm 18.06$ & 4.396 & 0.004 \\
\hline Serum uric acid (mg/dl) & $3.83 \pm 1.14$ & $4.06 \pm 1.12$ & $4.30 \pm 1.14$ & $4.62 \pm 1.22$ & 21.304 & $<0.001$ \\
\hline Systolic blood pressure (mmHg) & $133.66 \pm 24.74$ & $136.74 \pm 23.69$ & $141.83 \pm 30.53$ & $146.33 \pm 38.15$ & 19.704 & $<0.001$ \\
\hline Diastolic blood pressure (mmHg) & $73.00 \pm 12.26$ & $75.01 \pm 11.77$ & $78.61 \pm 12.06$ & $79.54 \pm 12.23$ & 10.742 & $<0.001$ \\
\hline Body mass index $\left(\mathrm{kg} / \mathrm{m}^{2}\right)$ & $17.13 \pm 1.42$ & $21.43 \pm 1.50$ & $25.73 \pm 1.15$ & $30.90 \pm 4.33$ & 2202.200 & $<0.001$ \\
\hline Triglycerides (mg/dl) & $103.17 \pm 50.81$ & $128.46 \pm 82.33$ & $158.53 \pm 100.30$ & $176.54 \pm 128.24$ & 33.113 & $<0.001$ \\
\hline
\end{tabular}




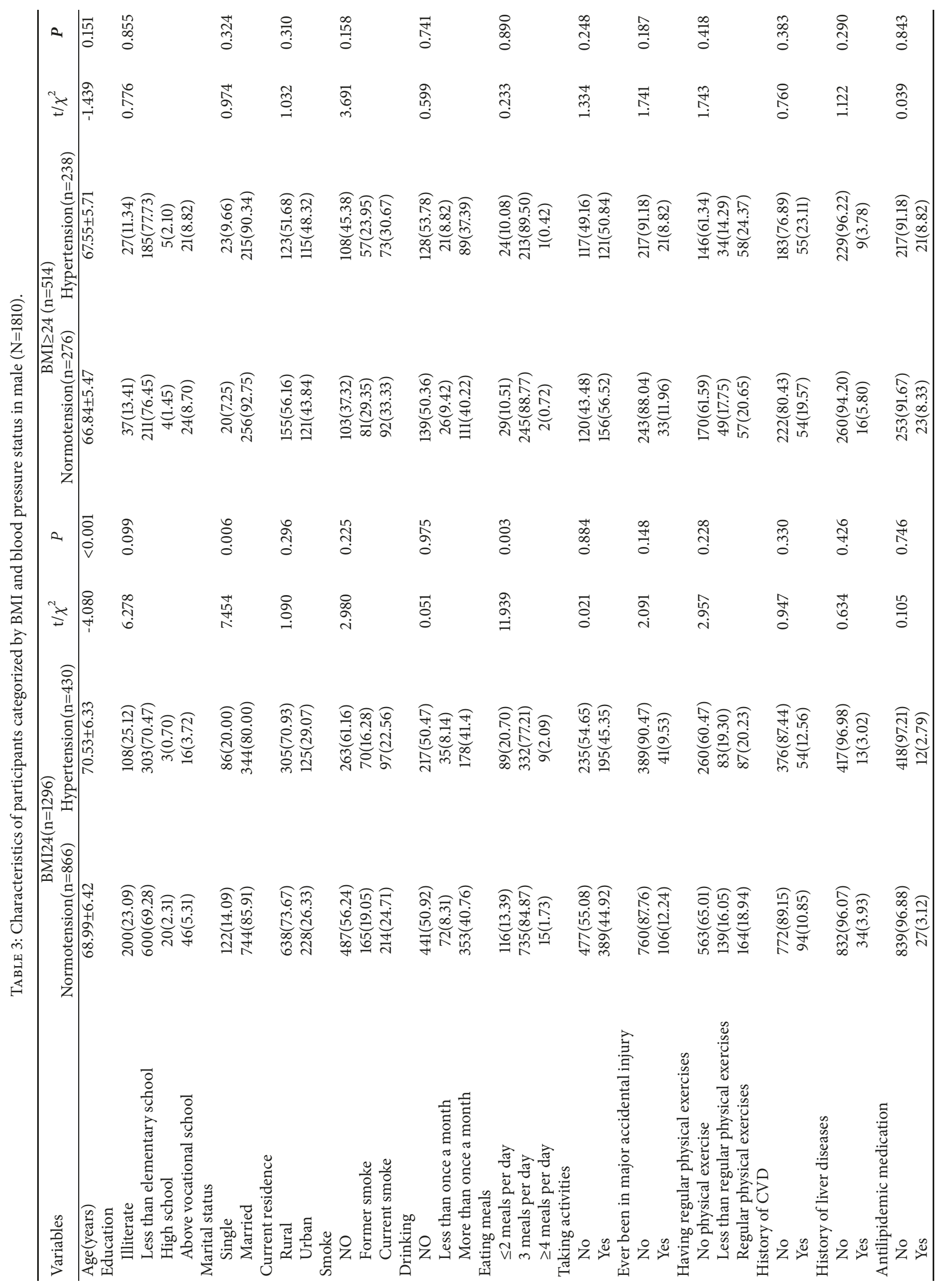




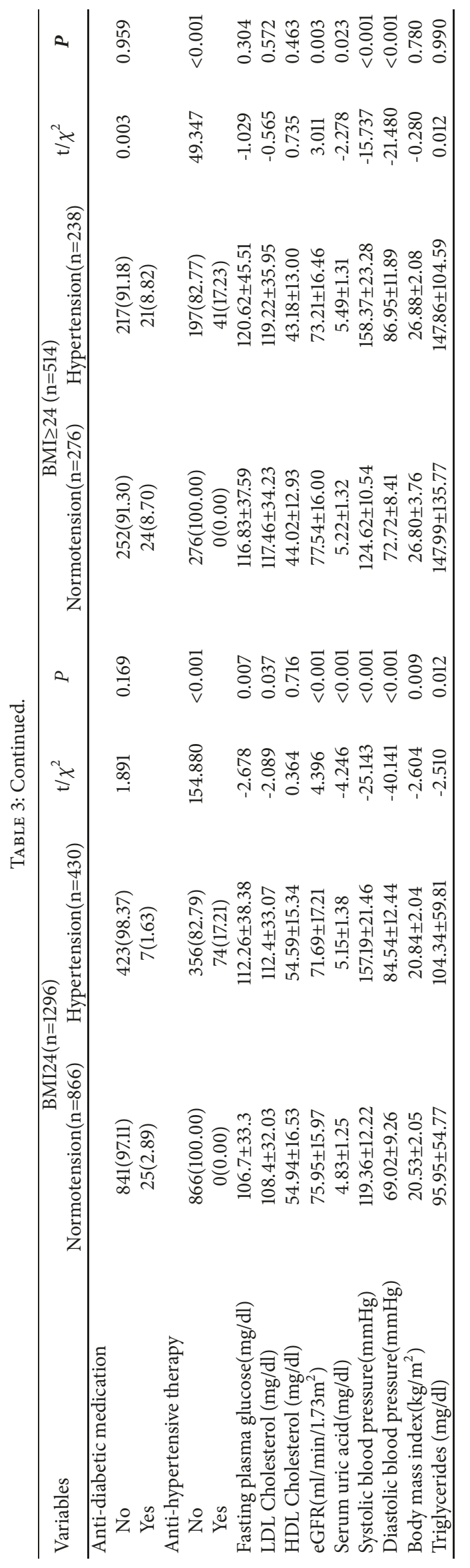




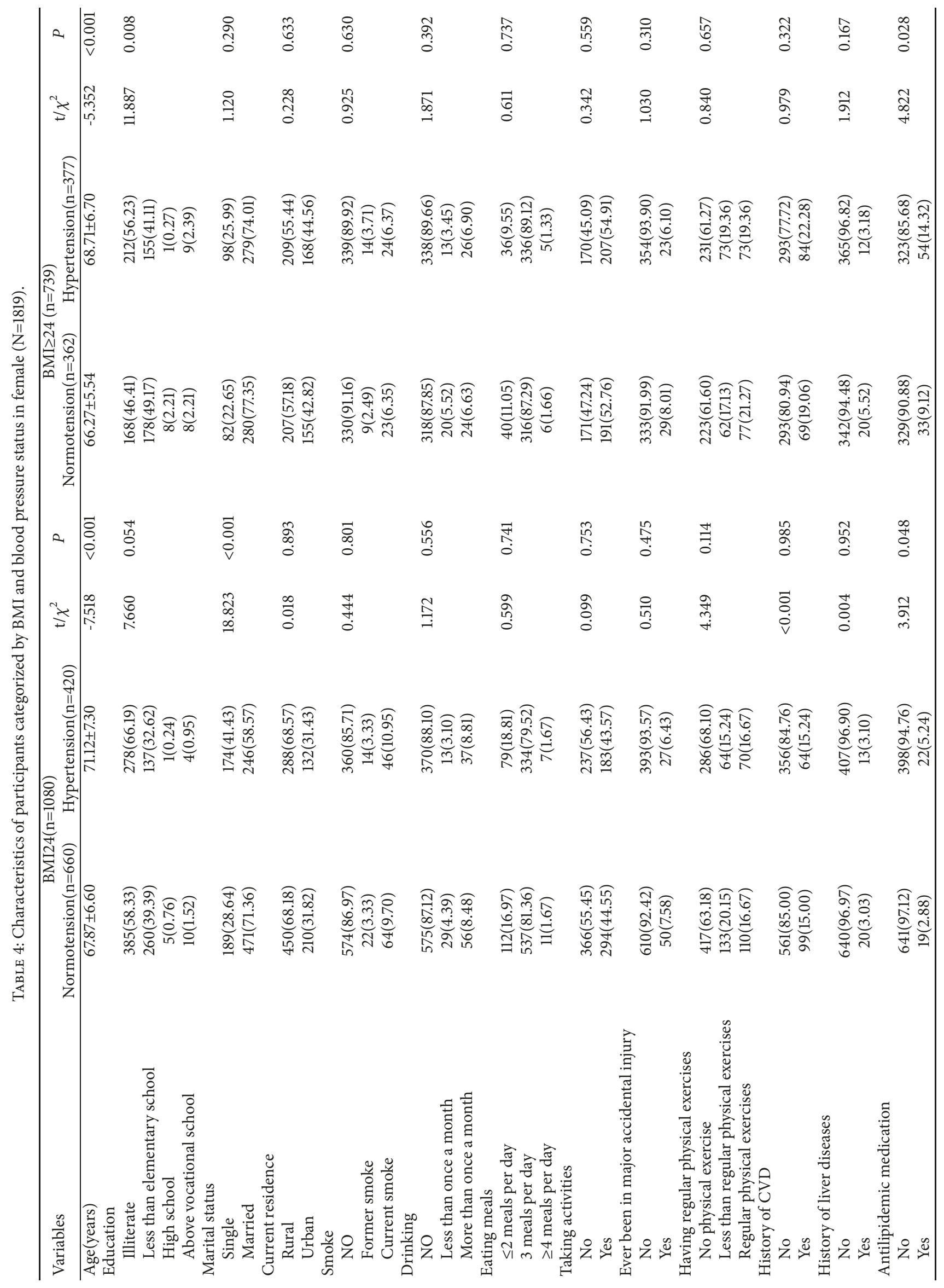




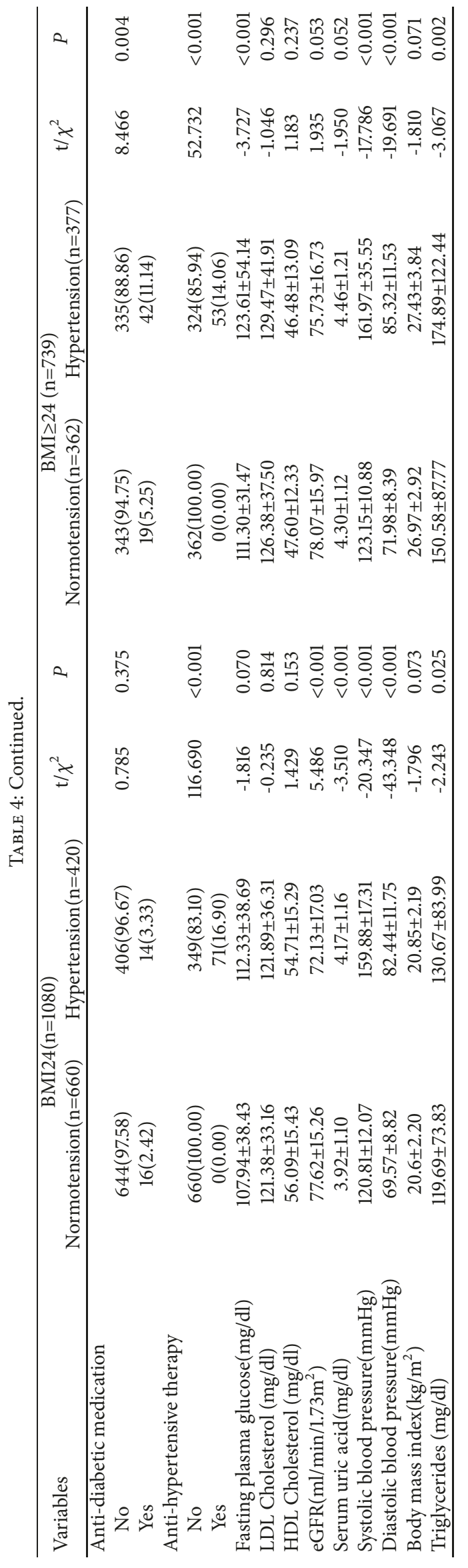




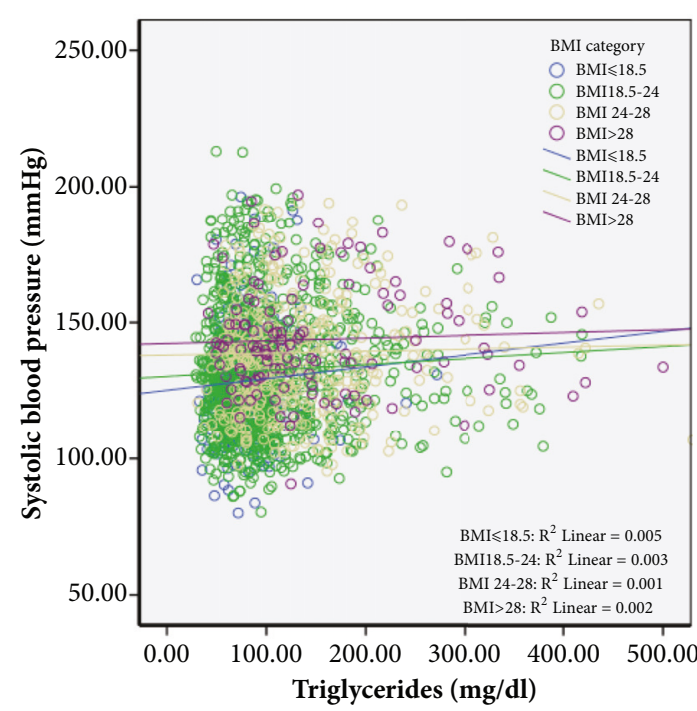

(a)

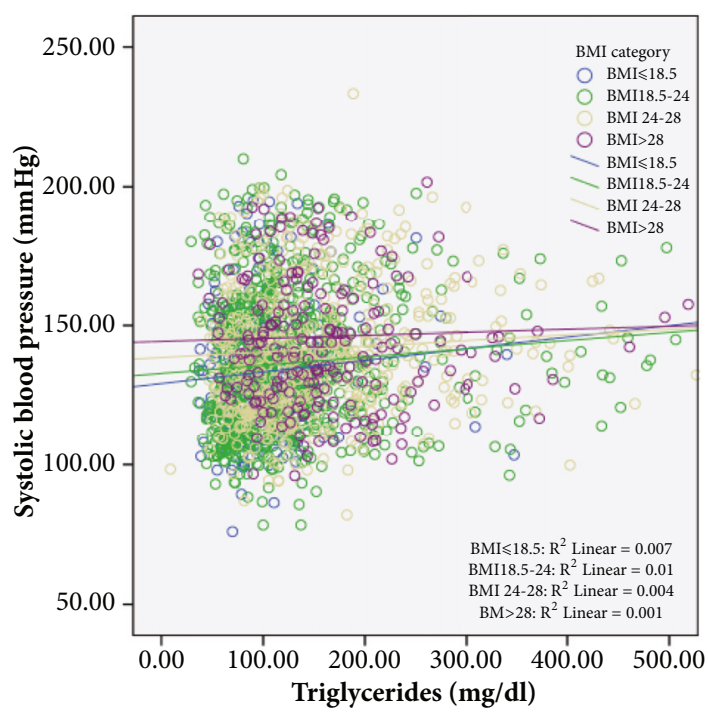

(c)

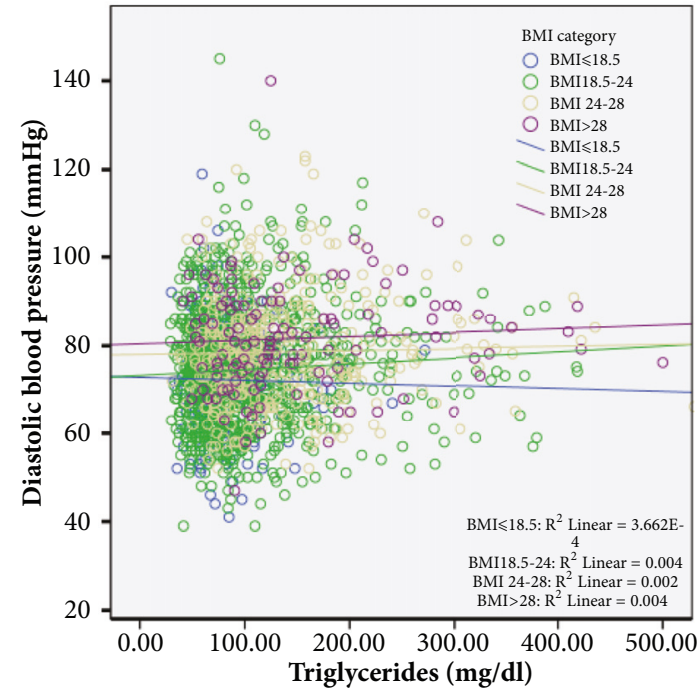

(b)

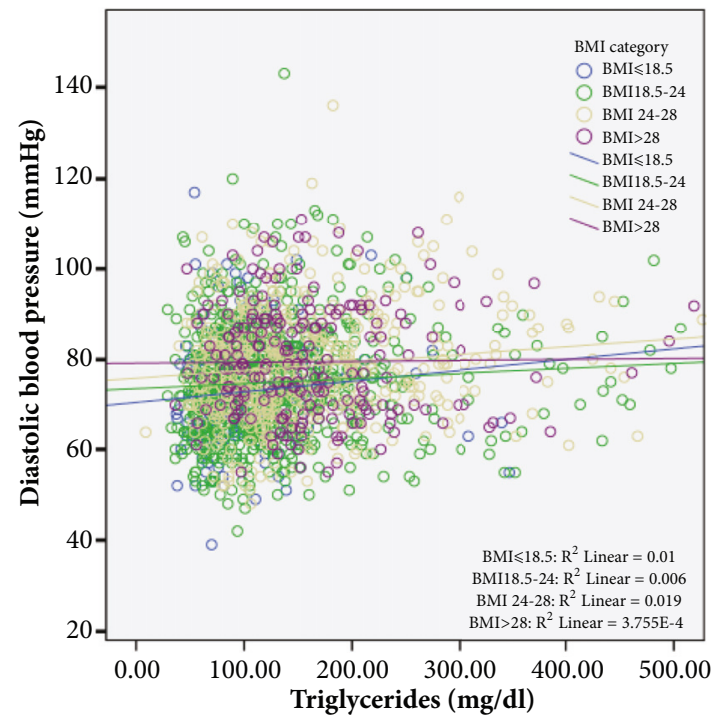

(d)

Figure 1: ( $a, b)$ Correlation between serum uric acid and blood pressure status of participants categorized by body mass index in male. (c, d) Correlation between triglycerides and blood pressure status of participants categorized by body mass index in female.

Second, in the hypertensive group with a BMI $\geq 24.0 \mathrm{~kg} / \mathrm{m}^{2}$ in men, levels of SUA, SBP, and DBP were also significantly higher, but eGFR level was significantly lower than that in the normotensive group (shown in Table 3). Third, in the hypertensive group with a BMI $<24.0 \mathrm{~kg} / \mathrm{m}^{2}$ in women, levels of TG, SUA, SBP, DBP, and the prevalence of antilipidemic medication were significantly higher than those in the normotensive group, but eGFR level was significantly lower. Lastly, in the hypertensive group with a BMI $\geq 24.0 \mathrm{~kg} / \mathrm{m}^{2}$ in women, the prevalence of CVD, antilipidemic medication, and antidiabetic medication were significantly higher, as were levels of glucose, TG, SBP, and DBP (Table 4).

Table 5 shows the various characteristics of participants categorized by age. SUA and SBP levels were significantly higher in the older age group than in the younger age group in men, whereas levels of HDL-C, eGFR, DBP, BMI, and
TG were lower in the older age group than in the younger age group. However, between-group differences in categories of current residence, eating habits, taking activities, major accidental injury, regular physical exercise, history of liver disease, antidiabetic medication, antihypertensive therapy, FPG, and LDL-C were not observed. In women, SUA and SBP levels were significantly higher in the older age group than in the younger age group, but eGFR and BMI levels were lower in the older age group than in the younger age group. However, between-group differences in categories of current residence, drinking, eating habits, activity, major accidental injury, history of CVD, history of liver disease, antidiabetic medication, and antihypertensive therapy, FPG, LDL-C, HDL-C, DBP, and TG were not observed.

In addition to their direct associations, we observed the effect between BMI category and TG levels on BP in Figure 1. 


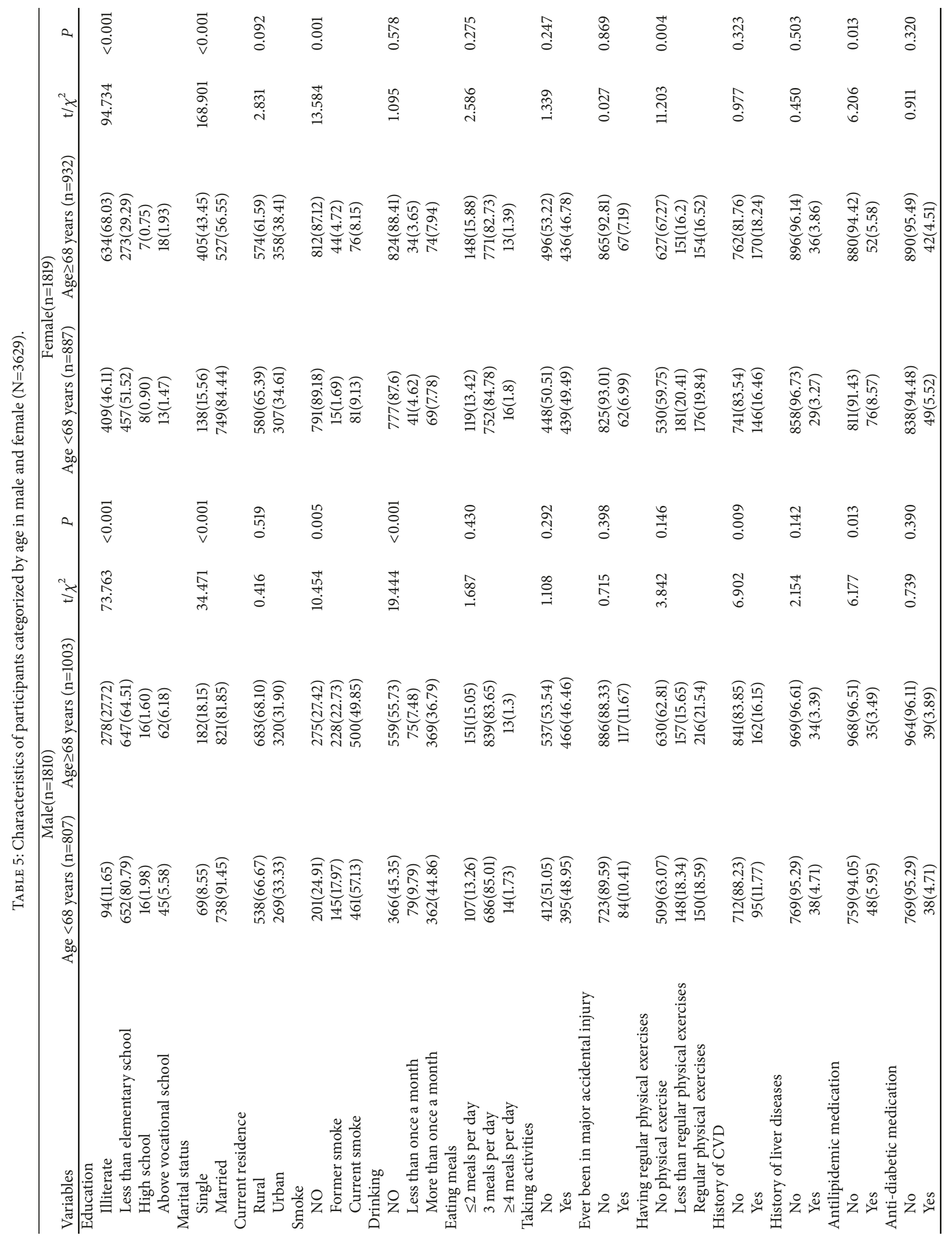




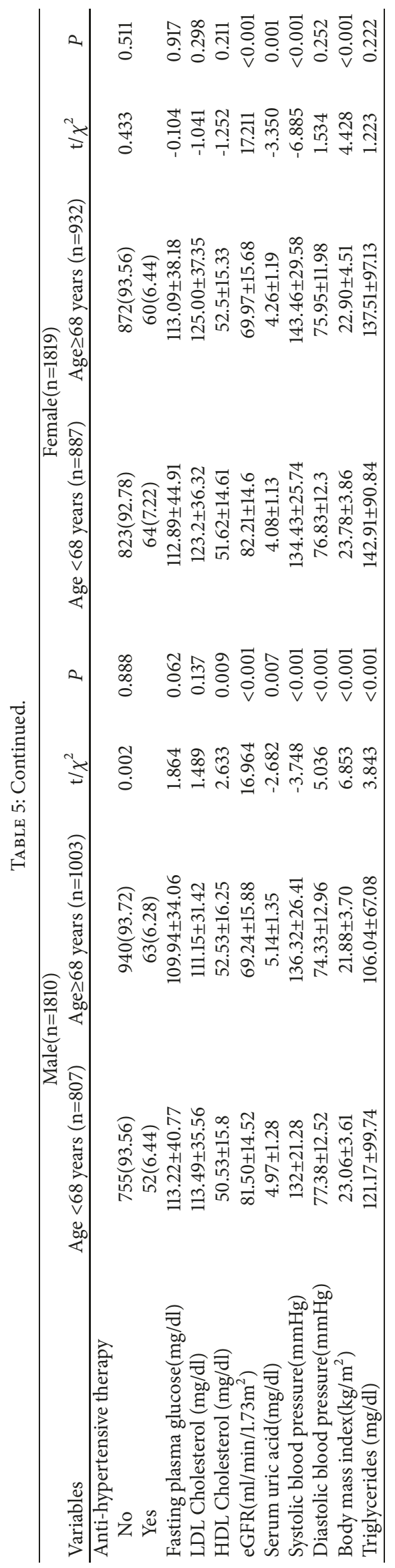


TABLE 6: Age-adjusted relationship between various characteristics and blood pressure status of participants categorized by BMI in male $(\mathrm{N}=1810)$.

\begin{tabular}{|c|c|c|c|c|}
\hline \multirow[b]{2}{*}{ Variables } & \multicolumn{2}{|c|}{$\mathrm{BMI}<24(\mathrm{n}=1296)$} & \multicolumn{2}{|c|}{$\mathrm{BMI} \geq 24(\mathrm{n}=514)$} \\
\hline & $\begin{array}{l}\text { Systolic blood pressure } \\
\text { partial } \mathrm{r}(P \text {-value })\end{array}$ & $\begin{array}{l}\text { Diastolic blood pressure } \\
\text { partial } \mathrm{r}(P \text {-value })\end{array}$ & $\begin{array}{l}\text { Systolic blood pressure } \\
\text { partial } \mathrm{r}(P \text {-value })\end{array}$ & $\begin{array}{l}\text { Diastolic blood pressure } \\
\text { partial } \mathrm{r}(P \text {-value })\end{array}$ \\
\hline $\begin{array}{l}\text { Education }(0=\text { Illiterate, } 1=\text { Less } \\
\text { than elementary school, } 2=\text { High } \\
\text { school, } 3=\text { Above vocational } \\
\text { school })\end{array}$ & $-0.024(0.392)$ & $0.013(0.647)$ & $-0.009(0.848)$ & $-0.042(0.344)$ \\
\hline $\begin{array}{l}\text { Marital status }(0=\text { Single }, 1= \\
\text { Married })\end{array}$ & $-0.105(<0.001)$ & $-0.092(0.001)$ & $0.011(0.807)$ & $-0.011(0.798)$ \\
\hline $\begin{array}{l}\text { Current residence }(0=\text { Rural, } 1= \\
\text { Urban })\end{array}$ & $0.044(0.117)$ & $0.032(0.249)$ & $0.113(0.011)$ & $0.059(0.187)$ \\
\hline $\begin{array}{l}\text { Smoke }(0=\text { NO, } 1=\text { Former } \\
\text { smoke, } 2=\text { Current smoke })\end{array}$ & $0.06(0.033)$ & $0.009(0.752)$ & $0.066(0.140)$ & $0.016(0.727)$ \\
\hline $\begin{array}{l}\operatorname{Drinking}(0=\mathrm{NO}, 1=\text { Less than } \\
\text { once a month, } 2=\text { More than once } \\
\text { a month })\end{array}$ & $0.015(0.602)$ & $0.020(0.480)$ & $-0.066(0.139)$ & $-0.003(0.944)$ \\
\hline $\begin{array}{l}\text { Eating meals }(0=\leq 2 \text { meals per } \\
\text { day, } 1=3 \text { meals per day, } 2=\geq 4 \\
\text { meals per day })\end{array}$ & $-0.061(0.031)$ & $-0.083(0.003)$ & $-0.014(0.759)$ & $-0.056(0.21)$ \\
\hline Taking activities $(0=$ No, $1=$ Yes $)$ & $0.014(0.623)$ & $0.031(0.268)$ & $0.091(0.040)$ & $0.118(0.008)$ \\
\hline $\begin{array}{l}\text { Ever been in major accidental } \\
\text { injury }(0=\text { No, } 1=\text { Yes })\end{array}$ & $-0.028(0.320)$ & $-0.019(0.505)$ & $-0.035(0.429)$ & $-0.007(0.871)$ \\
\hline $\begin{array}{l}\text { Having regular physical } \\
\text { exercises }(0=\text { No physical } \\
\text { exercise, } 1=\text { Less than regular } \\
\text { physical exercises, } 2=\text { Regular } \\
\text { physical exercises })\end{array}$ & $0.034(0.23)$ & $0.006(0.833)$ & $0.011(0.803)$ & $0.011(0.814)$ \\
\hline History of $\operatorname{CVD}(0=$ No, $1=$ Yes $)$ & $0.019(0.497)$ & $0.028(0.327)$ & $-0.009(0.833)$ & $0.008(0.854)$ \\
\hline $\begin{array}{l}\text { History of liver diseases }(0= \\
\text { No,1= Yes })\end{array}$ & $0.009(0.760)$ & $0.013(0.631)$ & $-0.023(0.613)$ & $0.028(0.526)$ \\
\hline $\begin{array}{l}\text { Antilipidemic medication }(0= \\
\text { No, } 1=\text { Yes })\end{array}$ & $-0.008(0.787)$ & $0.019(0.504)$ & $0.002(0.973)$ & $0.027(0.546)$ \\
\hline $\begin{array}{l}\text { Anti-diabetic medication }(0= \\
\text { No,1=Yes })\end{array}$ & $0.002(0.955)$ & $0.007(0.806)$ & $0.043(0.339)$ & $-0.012(0.796)$ \\
\hline $\begin{array}{l}\text { Anti-hypertensive therapy }(0= \\
\text { No,1=Yes })\end{array}$ & $0.140(<0.001)$ & $0.109(<0.001)$ & $0.047(0.291)$ & $0.127(0.004)$ \\
\hline Fasting plasma glucose (mg/dl) & $0.075(0.007)$ & $0.046(0.098)$ & $0.068(0.126)$ & $0.065(0.147)$ \\
\hline LDL Cholesterol (mg/dl) & $0.063(0.024)$ & $0.052(0.067)$ & $0.063(0.157)$ & $0.024(0.585)$ \\
\hline HDL Cholesterol (mg/dl) & $-0.005(0.848)$ & $0.005(0.870)$ & $-0.042(0.344)$ & $-0.003(0.942)$ \\
\hline $\mathrm{eGFR}\left(\mathrm{ml} / \mathrm{min} / 1.73 \mathrm{~m}^{2}\right)$ & $-0.076(0.007)$ & $-0.043(0.124)$ & $-0.157(<0.001)$ & $-0.107(0.016)$ \\
\hline Serum uric $\operatorname{acid}(\mathrm{mg} / \mathrm{dl})$ & $0.108(<0.001)$ & $0.06(0.032)$ & $0.056(0.212)$ & $0.067(0.130)$ \\
\hline Body mass index $\left(\mathrm{kg} / \mathrm{m}^{2}\right)$ & $0.121(<0.001)$ & $0.093(0.001)$ & $0.062(0.163)$ & $0.048(0.279)$ \\
\hline Triglycerides (mg/dl) & $0.069(0.014)$ & $0.058(0.04)$ & $0.074(0.097)$ & $0.047(0.288)$ \\
\hline
\end{tabular}

TG correlated positively with both SBP and DBP. Analysis of covariance showed that three regression lines in each graph were not different from those in the other groups (male: SBP, $\mathrm{F}=0.028, P=0.867$ and $\mathrm{DBP}, \mathrm{F}=0.194$, and $P=0.660$; female: SBP, $\mathrm{F}=0.783, P=0.376$ and $\mathrm{DBP}, \mathrm{F}=0.005$, and $P=0.941$; respectively).

Tables 6 and 7 show the relationship between various characteristics and BP status of participants categorized by BMI $\left(<24.0 \mathrm{~kg} / \mathrm{m}^{2}\right.$ and $\left.\geq 24.0 \mathrm{~kg} / \mathrm{m}^{2}\right)$. Age-adjusted partial
Pearson's correlation coefficient showed that TG level was positively correlated with both SBP and DBP in men with a BMI $<21.0 \mathrm{~kg} / \mathrm{m}^{2}$ (Table 6). In women, the TG level was significantly correlated with SBP and DBP in subjects with a $\mathrm{BMI}<21.0 \mathrm{~kg} / \mathrm{m}^{2}$ and positively correlated with DBP in those with a BMI $\geq 24.0 \mathrm{~kg} / \mathrm{m}^{2}$, but there was no correlation with $\mathrm{SBP}$ in women with a $\mathrm{BMI} \geq 24.0 \mathrm{~kg} / \mathrm{m}^{2}$ (Table 7 ).

Tables 8 and 9 show the relationship between various characteristics and BP status of participants categorized by 
TABLE 7: Age-adjusted relationship between various characteristics and blood pressure status of participants categorized by BMI in female $(\mathrm{N}=1819)$.

\begin{tabular}{|c|c|c|c|c|}
\hline \multirow[b]{2}{*}{ Variables } & \multicolumn{2}{|c|}{$\mathrm{BMI}<24(\mathbf{n}=\mathbf{1 0 8 0})$} & \multicolumn{2}{|c|}{$\mathrm{BMI} \geq 24(\mathbf{n}=739)$} \\
\hline & $\begin{array}{l}\text { Systolic blood pressure } \\
\text { partial } \mathrm{r}(P \text {-value })\end{array}$ & $\begin{array}{l}\text { Diastolic blood pressure } \\
\text { partial } \mathrm{r}(P \text {-value })\end{array}$ & $\begin{array}{l}\text { Systolic blood pressure } \\
\text { partial } \mathrm{r}(P \text {-value })\end{array}$ & $\begin{array}{l}\text { Diastolic blood pressure } \\
\text { partial } \mathrm{r}(P \text {-value })\end{array}$ \\
\hline $\begin{array}{l}\text { Education }(0=\text { Illiterate, } 1=\text { Less } \\
\text { than elementary school, } 2=\text { High } \\
\text { school, } 3=\text { Above vocational } \\
\text { school })\end{array}$ & $-0.053(0.086)$ & $-0.07(0.024)$ & $-0.002(0.957)$ & $-0.041(0.270)$ \\
\hline $\begin{array}{l}\text { Marital status }(0=\text { Single }, 1= \\
\text { Married })\end{array}$ & $-0.097(0.002)$ & $-0.077(0.012)$ & $-0.066(0.076)$ & $-0.015(0.687)$ \\
\hline $\begin{array}{l}\text { Current residence }(0=\text { Rural, } 1= \\
\text { Urban })\end{array}$ & $-0.01(0.745)$ & $-0.039(0.211)$ & $0.039(0.294)$ & $0.035(0.344)$ \\
\hline $\begin{array}{l}\text { Smoke }(0=\text { NO, } 1=\text { Former } \\
\text { smoke } 2=\text { Current smoke })\end{array}$ & $0.035(0.255)$ & $0.021(0.503)$ & $-0.025(0.508)$ & $0.007(0.844)$ \\
\hline $\begin{array}{l}\operatorname{Drinking}(0=\mathrm{NO}, 1=\text { Less than } \\
\text { once a month, } 2=\text { More than once } \\
\text { a month })\end{array}$ & $-0.016(0.608)$ & $0.015(0.630)$ & $-0.022(0.549)$ & $-0.02(0.584)$ \\
\hline $\begin{array}{l}\text { Eating meals }(0=\leq 2 \text { meals per } \\
\text { day, } 1=3 \text { meals per day, } 2=\geq 4 \\
\text { meals per day })\end{array}$ & $-0.021(0.505)$ & $-0.075(0.015)$ & $-0.026(0.483)$ & $-0.069(0.064)$ \\
\hline Taking activities $(0=$ No, $1=$ Yes $)$ & $-0.036(0.251)$ & $-0.034(0.266)$ & $0.027(0.474)$ & $0.027(0.472)$ \\
\hline $\begin{array}{l}\text { Ever been in major accidental } \\
\text { injury }(0=\text { No, } 1=\text { Yes })\end{array}$ & $-0.01(0.745)$ & $0.014(0.640)$ & $-0.041(0.274)$ & $-0.042(0.259)$ \\
\hline $\begin{array}{l}\text { Having regular physical } \\
\text { exercises }(0=\text { No physical } \\
\text { exercise, } 1=\text { Less than regular } \\
\text { physical exercises, } 2=\text { Regular } \\
\text { physical exercises })\end{array}$ & $-0.008(0.799)$ & $-0.008(0.803)$ & $-0.015(0.692)$ & $0.031(0.401)$ \\
\hline History of $\operatorname{CVD}(0=$ No, $1=$ Yes $)$ & $0.036(0.239)$ & $0.001(0.986)$ & $-0.014(0.700)$ & $-0.021(0.582)$ \\
\hline $\begin{array}{l}\text { History of liver diseases }(0= \\
\text { No,1= Yes })\end{array}$ & $0.005(0.868)$ & $0.003(0.934)$ & $-0.047(0.213)$ & $-0.103(0.006)$ \\
\hline $\begin{array}{l}\text { Antilipidemic medication }(0= \\
\text { No, } 1=\text { Yes })\end{array}$ & $0.065(0.034)$ & $0.054(0.082)$ & $0.054(0.151)$ & $0.032(0.399)$ \\
\hline $\begin{array}{l}\text { Anti-diabetic medication }(0= \\
\text { No,1=Yes })\end{array}$ & $0.013(0.686)$ & $-0.029(0.356)$ & $0.122(0.001)$ & $0.046(0.214)$ \\
\hline $\begin{array}{l}\text { Anti-hypertensive therapy }(0= \\
\text { No, } 1=\text { Yes })\end{array}$ & $0.110(<0.001)$ & $0.037(0.029)$ & $0.128(<0.001)$ & $0.102(<0.001)$ \\
\hline Fasting plasma glucose $(\mathrm{mg} / \mathrm{dl})$ & $0.049(0.115)$ & $0.043(0.161)$ & $0.021(0.577)$ & $0.158(<0.001)$ \\
\hline LDL Cholesterol (mg/dl) & $0.028(0.362)$ & $0.011(0.725)$ & $0.049(0.189)$ & $-0.005(0.886)$ \\
\hline HDL Cholesterol (mg/dl) & $-0.087(0.005)$ & $-0.06(0.051)$ & $-0.039(0.293)$ & $-0.050(0.184)$ \\
\hline $\mathrm{eGFR}\left(\mathrm{ml} / \mathrm{min} / 1.73 \mathrm{~m}^{2}\right)$ & $-0.069(0.025)$ & $-0.045(0.147)$ & $0.067(0.075)$ & $0.020(0.600)$ \\
\hline Serum uric $\operatorname{acid}(\mathrm{mg} / \mathrm{dl})$ & $0.126(<0.001)$ & $0.074(0.016)$ & $-0.018(0.627)$ & $0.011(0.764)$ \\
\hline Body mass index $\left(\mathrm{kg} / \mathrm{m}^{2}\right)$ & $0.105(0.001)$ & $0.093(0.002)$ & $0.021(0.583)$ & $0.075(0.045)$ \\
\hline Triglycerides (mg/dl) & $0.123(<0.001)$ & $0.091(0.003)$ & $0.046(0.218)$ & $0.097(0.010)$ \\
\hline
\end{tabular}

age ( $<68$ years and $\geq 68$ years). TG level was positively correlated with both SBP and DBP in both men and women with age $<68$ years and $\geq 68$ years.

Tables 10 and 11 show the multivariate-adjusted relationship between various characteristics and BP status in participants categorized by BMI $\left(<24.0 \mathrm{~kg} / \mathrm{m}^{2}\right.$ and $\geq 24.0$ $\mathrm{kg} / \mathrm{m}^{2}$ ). Multiple linear regression analysis showed that TG level was significantly and positively associated with SBP in women with a BMI $<24.0 \mathrm{~kg} / \mathrm{m}^{2}$, independent of other confounding factors; however, TG level was not significantly associated with BP in men.

Tables 12 and 13 show the multivariate-adjusted relationship between various characteristics and BP status in participants categorized by age ( $<68$ years and $\geq 68$ years). Multiple linear regression analysis showed that TG level was significantly and positively associated with SBP in women with age of $<68$ years, independent of other confounding factors. In contrast, TG level was significantly associated with SBP in men with age of $\geq 68$ years. 
TABLE 8: Relationship between various characteristics and blood pressure status of participants categorized by age in male ( $\mathrm{N}=1810$ ).

\begin{tabular}{|c|c|c|c|c|}
\hline \multirow{2}{*}{ Variables } & \multicolumn{2}{|c|}{$\begin{array}{c}\text { Age }<68 \text { years } \\
\quad(\mathrm{n}=807)\end{array}$} & \multicolumn{2}{|c|}{$\begin{array}{l}\text { Age } \geq 68 \text { years } \\
(\mathrm{n}=1003)\end{array}$} \\
\hline & $\begin{array}{l}\text { Systolic blood pressure } \\
\text { partial } \mathrm{r}(P \text {-value })\end{array}$ & $\begin{array}{l}\text { Diastolic blood pressure } \\
\text { partial } \mathrm{r}(P \text {-value })\end{array}$ & $\begin{array}{l}\text { Systolic blood pressure } \\
\text { partial } \mathrm{r}(P \text {-value })\end{array}$ & $\begin{array}{l}\text { Diastolic blood pressure } \\
\text { partial } \mathrm{r}(P \text {-value })\end{array}$ \\
\hline Age & $0.052(0.146)$ & $-0.011(0.763)$ & $0.05(0.116)$ & $-0.096(0.002)$ \\
\hline $\begin{array}{l}\text { Education }(0=\text { Illiterate, } 1=\text { Less } \\
\text { than elementary school, } 2=\text { High } \\
\text { school, } 3=\text { Above vocational } \\
\text { school })\end{array}$ & $0.013(0.720)$ & $-0.016(0.646)$ & $-0.019(0.544)$ & $0.043(0.173)$ \\
\hline $\begin{array}{l}\text { Marital status }(0=\text { Single }, 1= \\
\text { Married })\end{array}$ & $-0.064(0.070)$ & $-0.086(0.015)$ & $-0.074(0.020)$ & $-0.033(0.295)$ \\
\hline $\begin{array}{l}\text { Current residence }(0=\text { Rural }, 1= \\
\text { Urban })\end{array}$ & $0.072(0.042)$ & $0.039(0.274)$ & $0.102(0.001)$ & $0.096(0.003)$ \\
\hline $\begin{array}{l}\text { Smoke }(0=\text { NO, } 1=\text { Former } \\
\text { smoke, } 2=\text { Current smoke })\end{array}$ & $0.018(0.616)$ & $0.017(0.633)$ & $0.042(0.184)$ & $-0.023(0.462)$ \\
\hline $\begin{array}{l}\operatorname{Drinking}(0=\mathrm{NO}, 1=\text { Less than } \\
\text { once a month, } 2=\text { More than once } \\
\text { a month })\end{array}$ & $-0.028(0.425)$ & $-0.015(0.679)$ & $-0.006(0.856)$ & $0.037(0.244)$ \\
\hline $\begin{array}{l}\text { Eating meals }(0=\leq 2 \text { meals per } \\
\text { day, } 1=3 \text { meals per day, } 2=\geq 4 \\
\text { meals per day })\end{array}$ & $-0.065(0.067)$ & $-0.098(0.006)$ & $-0.026(0.420)$ & $-0.050(0.114)$ \\
\hline Taking activities $(0=$ No, $1=$ Yes $)$ & $-0.036(0.315)$ & $-0.040(0.255)$ & $0.022(0.495)$ & $0.042(0.189)$ \\
\hline $\begin{array}{l}\text { Ever been in major accidental } \\
\text { injury }(0=\text { No,l= Yes })\end{array}$ & $-0.044(0.212)$ & $-0.053(0.137)$ & $-0.026(0.416)$ & $-0.001(0.975)$ \\
\hline $\begin{array}{l}\text { Having regular physical } \\
\text { exercises }(0=\text { No physical } \\
\text { exercise, } 1=\text { Less than regular } \\
\text { physical exercises, } 2=\text { Regular } \\
\text { physical exercises })\end{array}$ & $-0.001(0.987)$ & $-0.005(0.89)$ & $0.046(0.147)$ & $0.022(0.489)$ \\
\hline History of $\operatorname{CVD}(0=$ No, $1=$ Yes $)$ & $0.055(0.123)$ & $0.06(0.089)$ & $0.009(0.786)$ & $0.027(0.396)$ \\
\hline $\begin{array}{l}\text { History of liver diseases }(0= \\
\text { No, } 1=\text { Yes })\end{array}$ & $-0.033(0.357)$ & $-0.019(0.598)$ & $0.024(0.448)$ & $0.056(0.078)$ \\
\hline $\begin{array}{l}\text { Antilipidemic medication }(0= \\
\text { No, } 1=\text { Yes })\end{array}$ & $0.060(0.092)$ & $0.06(0.088)$ & $-0.018(0.561)$ & $0.021(0.502)$ \\
\hline $\begin{array}{l}\text { Anti-diabetic medication }(0= \\
\text { No, } 1=\text { Yes })\end{array}$ & $0.038(0.281)$ & $-0.002(0.945)$ & $0.041(0.198)$ & $0.045(0.157)$ \\
\hline $\begin{array}{l}\text { Anti-hypertensive therapy }(0= \\
\text { No,1= Yes })\end{array}$ & $0.131(<0.001)$ & $0.122(0.001)$ & $0.103(0.001)$ & $0.118(<0.001)$ \\
\hline Fasting plasma glucose $(\mathrm{mg} / \mathrm{dl})$ & $0.122(0.001)$ & $0.092(0.009)$ & $0.069(0.031)$ & $0.048(0.131)$ \\
\hline LDL Cholesterol (mg/dl) & $0.096(0.007)$ & $0.067(0.056)$ & $0.061(0.054)$ & $0.054(0.087)$ \\
\hline HDL Cholesterol (mg/dl) & $-0.102(0.004)$ & $-0.077(0.028)$ & $-0.049(0.122)$ & $-0.026(0.405)$ \\
\hline $\mathrm{eGFR}\left(\mathrm{ml} / \mathrm{min} / 1.73 \mathrm{~m}^{2}\right)$ & $-0.119(0.001)$ & $-0.084(0.017)$ & $-0.117(<0.001)$ & $-0.016(0.618)$ \\
\hline Serum uric acid(mg/dl) & $0.143(<0.001)$ & $0.122(0.001)$ & $0.104(0.001)$ & $0.049(0.123)$ \\
\hline Body mass index $\left(\mathrm{kg} / \mathrm{m}^{2}\right)$ & $0.252(<0.001)$ & $0.218(<0.001)$ & $0.146(<0.001)$ & $0.140(<0.001)$ \\
\hline Triglycerides (mg/dl) & $0.091(0.010)$ & $0.091(0.010)$ & $0.105(0.001)$ & $0.083(0.009)$ \\
\hline
\end{tabular}

Table 14 shows the interaction between BMI and TG level and BP status in men and women. A general linear model with the following confounding factors (age, educational level, marital status, current residence, smoking, eating habits, taking activities, antidiabetic medication, antihypertensive therapy, FPG, LDL-C, eGFR, and SUA) was used to assess the statistical significance of the synergistic relationship between BMI and SUA level. Evidence of interaction between BMI and SUA level on SBP (men, $\beta=0.572, P=$
0.845 ; women, $\beta=0.122$, and $P=0.923$ ) and DBP (men, $\beta$ $=-0.373, P=0.810$; women, $\beta=0.272$, and $P=0.828$ ) levels was not observed.

Table 15 shows the interaction between BMI and TG on BP status of participants categorized by age in men and women. A general linear model with the following confounding factors (age, educational level, marital status, current residence, smoking, eating habits, activity, physical activity, antilipidemic medication, antidiabetic medication, 
TABLE 9: Relationship between various characteristics and blood pressure status of participants categorized by age in female ( $\mathrm{N}=1819)$.

\begin{tabular}{|c|c|c|c|c|}
\hline \multirow{2}{*}{ Variables } & \multicolumn{2}{|c|}{$\begin{array}{l}\text { Age }<68 \text { years } \\
\quad(\mathrm{n}=887)\end{array}$} & \multicolumn{2}{|c|}{$\begin{array}{l}\text { Age } \geq 68 \text { years } \\
\quad(\mathrm{n}=932)\end{array}$} \\
\hline & $\begin{array}{l}\text { Systolic blood pressure } \\
\text { partial } \mathrm{r}(P \text {-value })\end{array}$ & $\begin{array}{l}\text { Diastolic blood pressure } \\
\text { partial } \mathrm{r}(P \text {-value })\end{array}$ & $\begin{array}{l}\text { Systolic blood pressure } \\
\text { partial } \mathrm{r}(P \text {-value })\end{array}$ & $\begin{array}{l}\text { Diastolic blood pressure } \\
\text { partial } \mathrm{r}(P \text {-value })\end{array}$ \\
\hline Age & $0.067(0.048)$ & $-0.032(0.348)$ & $0.117(<0.001)$ & $-0.039(0.244)$ \\
\hline $\begin{array}{l}\text { Education }(0=\text { Illiterate, } 1=\text { Less } \\
\text { than elementary school, } 2=\text { High } \\
\text { school, } 3=\text { Above vocational } \\
\text { school })\end{array}$ & $-0.033(0.335)$ & $-0.020(0.546)$ & $-0.034(0.308)$ & $-0.051(0.124)$ \\
\hline $\begin{array}{l}\text { Marital status }(0=\text { Single }, 1= \\
\text { Married })\end{array}$ & $-0.073(0.031)$ & $-0.028(0.409)$ & $-0.104(0.002)$ & $-0.035(0.292)$ \\
\hline $\begin{array}{l}\text { Current residence }(0=\text { Rural, } 1= \\
\text { Urban })\end{array}$ & $0.034(0.320)$ & $0.028(0.410)$ & $0.021(0.536)$ & $-0.007(0.822)$ \\
\hline $\begin{array}{l}\text { Smoke }(0=\text { NO, } 1=\text { Former } \\
\text { smoke, } 2=\text { Current smoke })\end{array}$ & $0.043(0.203)$ & $0.024(0.481)$ & $-0.040(0.230)$ & $-0.024(0.471)$ \\
\hline $\begin{array}{l}\operatorname{Drinking}(0=N O, 1=\text { Less than } \\
\text { once a month, } 2=\text { More than once } \\
\text { a month })\end{array}$ & $-0.017(0.605)$ & $-0.008(0.818)$ & $-0.028(0.391)$ & $0.001(0.970)$ \\
\hline $\begin{array}{l}\text { Eating meals }(0=\leq 2 \text { meals per } \\
\text { day, } 1=3 \text { meals per day, } 2=\geq 4 \\
\text { meals per day })\end{array}$ & $-0.053(0.118)$ & $-0.07(0.039)$ & $0.013(0.700)$ & $-0.041(0.221)$ \\
\hline Taking activities $(0=$ No, $1=$ Yes $)$ & $0.022(0.509)$ & $0.053(0.115)$ & $-0.002(0.956)$ & $-0.049(0.142)$ \\
\hline $\begin{array}{l}\text { Ever been in major accidental } \\
\text { injury }(0=\text { No, } 1=\text { Yes })\end{array}$ & $-0.018(0.589)$ & $0.012(0.719)$ & $-0.034(0.300)$ & $-0.032(0.330)$ \\
\hline $\begin{array}{l}\text { Having regular physical } \\
\text { exercises }(0=\text { No physical } \\
\text { exercise, } 1=\text { Less than regular } \\
\text { physical exercises, } 2=\text { Regular } \\
\text { physical exercises })\end{array}$ & $-0.016(0.638)$ & $0.025(0.460)$ & $-0.011(0.735)$ & $0.007(0.834)$ \\
\hline History of $\operatorname{CVD}(0=$ No, $1=$ Yes $)$ & $0.079(0.020)$ & $0.074(0.028)$ & $-0.032(0.332)$ & $-0.057(0.084)$ \\
\hline $\begin{array}{l}\text { History of liver diseases }(0= \\
\text { No,1= Yes })\end{array}$ & $0.019(0.574)$ & $0.011(0.746)$ & $-0.054(0.104)$ & $-0.088(0.008)$ \\
\hline $\begin{array}{l}\text { Antilipidemic medication }(0= \\
\text { No,1=Yes })\end{array}$ & $0.095(0.005)$ & $0.075(0.027)$ & $0.058(0.081)$ & $0.046(0.161)$ \\
\hline $\begin{array}{l}\text { Anti-diabetic medication }(0= \\
\text { No, } 1=\text { Yes })\end{array}$ & $0.125(<0.001)$ & $0.061(0.069)$ & $0.057(0.087)$ & $-0.004(0.896)$ \\
\hline $\begin{array}{l}\text { Anti-hypertensive therapy }(0= \\
\text { No,1=Yes })\end{array}$ & $0.096(0.004)$ & $0.060(0.073)$ & $0.135(<0.001)$ & $0.076(0.022)$ \\
\hline Fasting plasma glucose $(\mathrm{mg} / \mathrm{dl})$ & $0.061(0.072)$ & $0.159(<0.001)$ & $0.031(0.344)$ & $0.055(0.097)$ \\
\hline LDL Cholesterol (mg/dl) & $0.048(0.157)$ & $0.028(0.404)$ & $0.05(0.132)$ & $0.014(0.668)$ \\
\hline HDL Cholesterol (mg/dl) & $-0.137(<0.001)$ & $-0.118(<0.001)$ & $-0.063(0.058)$ & $-0.085(0.010)$ \\
\hline $\mathrm{eGFR}\left(\mathrm{ml} / \mathrm{min} / 1.73 \mathrm{~m}^{2}\right)$ & $-0.039(0.248)$ & $-0.007(0.836)$ & $-0.025(0.455)$ & $-0.011(0.743)$ \\
\hline Serum uric acid(mg/dl) & $0.084(0.012)$ & $0.083(0.013)$ & $0.092(0.005)$ & $0.054(0.100)$ \\
\hline Body mass index $\left(\mathrm{kg} / \mathrm{m}^{2}\right)$ & $0.161(<0.001)$ & $0.253(<0.001)$ & $0.116(<0.001)$ & $0.116(<0.001)$ \\
\hline Triglycerides (mg/dl) & $0.155(<0.001)$ & $0.148(<0.001)$ & $0.069(0.037)$ & $0.096(0.004)$ \\
\hline
\end{tabular}

antihypertensive therapy, FPG, eGFR, and SUA) was used to assess the statistical significance of the synergistic relationship between BMI and SUA level. Evidence of interaction between BMI and SUA level on SBP (men with age of $<68$ years, $\beta=-1.075$, and $P=0.760$; men with age of $\geq 68$ years, $\beta=2.138$, and $P=0.654$; women with age of $<68$ years, $\beta$ $=-1.345$, and $P=0.718$; women with age of $\geq 68$ years, $\beta=$ -4.192 , and $P=0.334$ ) and DBP (men with age of $<68$ years, $\beta=-0.095$, and $P=0.964$; men with age of $\geq 68$ years, $\beta=-$ 0.931 , and $P=0.691$; women with age of $<68$ years, $\beta=2.410$, $P=0.170$; women with age of $\geq 68$ years $\beta=-3.386$, and $P=$ $0.439)$ was not observed.

Table 16 shows relationships between BMI or TG level and hypertension in men and women. After adjusting for age, educational level, marital status, current residence, smoking, drinking, eating habits, activity, major accidental 
TABLE 10: Multivariate-adjusted relationship between various characteristics and blood pressure status of participants categorized by BMI in male $(\mathrm{N}=1810)$.

\begin{tabular}{|c|c|c|c|c|}
\hline \multirow[t]{2}{*}{ Variables } & \multicolumn{2}{|c|}{$\mathrm{BMI}<24(\mathrm{n}=1296)$} & \multicolumn{2}{|c|}{$\mathrm{BMI} \geq 24(\mathrm{n}=514)$} \\
\hline & $\begin{array}{l}\text { Systolic blood } \\
\text { pressure } \\
\beta(P \text {-value })\end{array}$ & $\begin{array}{l}\text { Diastolic blood } \\
\text { pressure } \\
\beta(P \text {-value })\end{array}$ & $\begin{array}{l}\text { Systolic blood } \\
\text { pressure } \\
\beta(P \text {-value })\end{array}$ & $\begin{array}{c}\text { Diastolic blood } \\
\text { pressure } \\
\beta(P \text {-value })\end{array}$ \\
\hline Age (years) & $0.117(<0.001)$ & $-0.108(0.001)$ & - & $-0.199(<0.001)$ \\
\hline $\begin{array}{l}\text { Education ( } 0=\text { Illiterate, } 1=\text { Less than } \\
\text { elementary school, } 2=\text { High school, } 3=\text { Above } \\
\text { vocational school) }\end{array}$ & - & - & - & - \\
\hline Marital status $(0=$ Single, $1=$ Married $)$ & $-0.101(<0.001)$ & $-0.092(0.001)$ & - & - \\
\hline Current residence $(0=$ Rural, $1=$ Urban $)$ & - & - & $0.119(0.009)$ & - \\
\hline $\begin{array}{l}\text { Smoke }(0=\text { NO, } 1=\text { Former smoke }, 2=\text { Current } \\
\text { smoke) }\end{array}$ & $0.060(0.033)$ & - & $0.104(0.023)$ & - \\
\hline $\begin{array}{l}\text { Drinking }(0=\mathrm{NO}, 1=\text { Less than once a } \\
\text { month, } 2=\text { More than once a month })\end{array}$ & - & - & - & - \\
\hline $\begin{array}{l}\text { Eating meals }(0=\leq 2 \text { meals per day, } 1=3 \text { meals } \\
\text { per day, } 2=\geq 4 \text { meals per day) }\end{array}$ & $-0.056(0.041)$ & $-0.085(0.002)$ & - & - \\
\hline Taking activities $(0=$ No, $1=$ Yes $)$ & - & - & $-0.108(0.016)$ & $-0.116(0.01)$ \\
\hline $\begin{array}{l}\text { Ever been in major accidental injury }(0=\text { No, } 1= \\
\text { Yes) }\end{array}$ & - & - & - & - \\
\hline $\begin{array}{l}\text { Having regular physical exercises }(0=\text { No } \\
\text { physical exercise, } 1=\text { Less than regular physical } \\
\text { exercises, } 2=\text { Regular physical exercises })\end{array}$ & - & - & - & - \\
\hline History of CVD $(0=$ No, $1=$ Yes $)$ & - & - & - & - \\
\hline History of liver diseases $(0=$ No, $1=$ Yes $)$ & - & - & - & - \\
\hline Antilipidemic medication $(0=\mathrm{No}, 1=\mathrm{Yes})$ & - & - & - & - \\
\hline Anti-diabetic medication $(0=$ No, $1=$ Yes $)$ & - & - & - & - \\
\hline Anti-hypertensive therapy $(0=$ No, $1=$ Yes $)$ & $0.130(<0.001)$ & $0.103(<0.001)$ & - & $0.113(0.011)$ \\
\hline Fasting plasma glucose (mg/dl) & $0.072(0.016)$ & - & - & - \\
\hline LDL Cholesterol (mg/dl) & $0.056(0.042)$ & - & $0.091(0.048)$ & - \\
\hline HDL Cholesterol (mg/dl) & - & - & - & - \\
\hline $\operatorname{eGFR}\left(\mathrm{ml} / \mathrm{min} / 1.73 \mathrm{~m}^{2}\right)$ & - & - & $-0.172(0.002)$ & - \\
\hline Serum uric acid (mg/dl) & $0.068(0.029)$ & - & - & - \\
\hline Body mass index $\left(\mathrm{kg} / \mathrm{m}^{2}\right)$ & $0.106(<0.001)$ & $0.076(0.009)$ & - & - \\
\hline Triglycerides (mg/dl) & - & - & - & - \\
\hline $\mathrm{R}^{2}$ & $0.098(<0.001)$ & $0.054(<0.001)$ & $0.087(0.003)$ & $0.089(0.0002)$ \\
\hline
\end{tabular}

injury, physical activity, history of CVD, history of liver disease, antilipidemic medication, antidiabetic medication, antihypertensive therapy, FPG, LDL-C, HDL-C, eGFR, and SUA, compared with their counterparts with BMI $<24.0$ $\mathrm{kg} / \mathrm{m}^{2}$, both elderly men and women with a BMI $\geq 24.0 \mathrm{~kg} / \mathrm{m}^{2}$ were more likely to have high $\mathrm{BP}$ (men: odds ratio [OR] = $1.781,95 \%$ confidence interval $[\mathrm{CI}]=1.393-2.277$; women: OR $=1.653,95 \% \mathrm{CI}=1.330-2.055)$. Among women, compared to those with TG $<150 \mathrm{mg} / \mathrm{dL}$, individuals with a high TG level were more likely to have high $\mathrm{BP}(\mathrm{OR}=1.558,95 \% \mathrm{CI}=$ 1.219-1.992).

Tables 17 and 18 show relationships between BMI or TG and hypertension categorized by age in men and women. After adjusting for age, educational level, marital status, current residence, smoking, drinking, eating habits, taking activities, major accidental injury, physical activity, history of CVD, history of liver disease, antilipidemic medication, antidiabetic medication, antihypertensive therapy, FPG, LDL-C, HDL-C, eGFR, and SUA, compared to individuals with a $\mathrm{BMI}<24.0 \mathrm{~kg} / \mathrm{m}^{2}$, both elderly men and women with a $\mathrm{BMI} \geq 24.0 \mathrm{~kg} / \mathrm{m}^{2}$ were more likely to have high BP (men with age of $<68$ years, $\mathrm{OR}=1.805$, and $95 \% \mathrm{CI}=1.249-2.610$; men with age of $\geq 68$ years, $\mathrm{OR}=1.796$, and $95 \% \mathrm{CI}=1.275-2.529$; women with age of $<68$ years, OR $=1.936$, and $95 \% \mathrm{CI}=$ 1.404-2.668; women with age of $\geq 68$ years, $\mathrm{OR}=1.506$, and $95 \% \mathrm{CI}=1.108-2.047)$. Among women, compared to those with TG $<150 \mathrm{mg} / \mathrm{dL}$, individuals with a high TG level were more likely to have high BP (women with age of $<68$ years, $\mathrm{OR}=1.629$, and $95 \% \mathrm{CI}=1.149-2.309$; women with age of $\geq$ 68 years, $\mathrm{OR}=1.596$, and $95 \% \mathrm{CI}=1.113-2.288)$. 
TABLE 11: Multivariate-adjusted relationship between various characteristics and blood pressure status of participants categorized by BMI in female $(\mathrm{N}=1819)$.

\begin{tabular}{|c|c|c|c|c|}
\hline \multirow[t]{2}{*}{ Variables } & \multicolumn{2}{|c|}{$\mathrm{BMI}<24(\mathrm{n}=1080)$} & \multicolumn{2}{|c|}{$\mathrm{BMI} \geq 24(\mathrm{n}=739)$} \\
\hline & $\begin{array}{l}\text { Systolic blood } \\
\text { pressure } \\
\beta(P \text {-value }) \\
\end{array}$ & $\begin{array}{l}\text { Diastolic blood } \\
\text { pressure } \\
\beta(P \text {-value })\end{array}$ & $\begin{array}{l}\text { Systolic blood } \\
\text { pressure } \\
\beta(P \text {-value })\end{array}$ & $\begin{array}{l}\text { Diastolic blood } \\
\text { pressure } \\
\beta(P \text {-value })\end{array}$ \\
\hline Age (years) & $0.168(<0.001)$ & - & $0.191(<0.001)$ & $0.191(<0.001)$ \\
\hline $\begin{array}{l}\text { Education ( } 0=\text { Illiterate, } 1=\text { Less than } \\
\text { elementary school, } 2=\text { High school, } 3=\text { Above } \\
\text { vocational school) }\end{array}$ & - & $-0.072(0.030)$ & - & - \\
\hline Marital status $(0=$ Single, $1=$ Married $)$ & $-0.106(0.001)$ & $-0.084(0.013)$ & $-0.082(0.034)$ & $-0.082(0.034)$ \\
\hline Current residence $(0=$ Rural, $1=$ Urban $)$ & - & - & - & - \\
\hline $\begin{array}{l}\text { Smoke }(0=\text { NO, } 1=\text { Former smoke }, 2=\text { Current } \\
\text { smoke) }\end{array}$ & - & - & - & - \\
\hline $\begin{array}{l}\text { Drinking }(0=\mathrm{NO}, 1=\text { Less than once a } \\
\text { month, } 2=\text { More than once a month })\end{array}$ & - & - & - & - \\
\hline $\begin{array}{l}\text { Eating meals }(0=\leq 2 \text { meals per day, } 1=3 \text { meals } \\
\text { per day, } 2=\geq 4 \text { meals per day) }\end{array}$ & - & $-0.075(0.016)$ & - & - \\
\hline Taking activities $(0=$ No, $1=$ Yes $)$ & - & - & - & - \\
\hline $\begin{array}{l}\text { Ever been in major accidental injury }(0=\text { No, } 1= \\
\text { Yes) }\end{array}$ & - & - & - & - \\
\hline $\begin{array}{l}\text { Having regular physical exercises }(0=\text { No } \\
\text { physical exercise, } 1=\text { Less than regular physical } \\
\text { exercises, } 2=\text { Regular physical exercises })\end{array}$ & - & - & - & - \\
\hline History of CVD ( $0=$ No,1= Yes $)$ & - & - & - & - \\
\hline History of liver diseases $(0=$ No, $1=$ Yes $)$ & - & - & - & - \\
\hline Antilipidemic medication $(0=$ No, $1=$ Yes $)$ & - & - & - & - \\
\hline Anti-diabetic medication $(0=$ No, $1=$ Yes $)$ & - & - & $0.138(0.001)$ & $0.138(0.001)$ \\
\hline Anti-hypertensive therapy $(0=$ No, $1=$ Yes $)$ & $0.12(<0.001)$ & - & $0.129(0.001)$ & $0.129(0.001)$ \\
\hline Fasting plasma glucose $(\mathrm{mg} / \mathrm{dl})$ & - & - & - & - \\
\hline LDL Cholesterol (mg/dl) & - & - & - & - \\
\hline HDL Cholesterol (mg/dl) & - & - & - & - \\
\hline $\mathrm{eGFR}\left(\mathrm{ml} / \mathrm{min} / 1.73 \mathrm{~m}^{2}\right)$ & - & - & - & - \\
\hline Serum uric $\operatorname{acid}(\mathrm{mg} / \mathrm{dl})$ & $0.084(0.016)$ & - & - & - \\
\hline Body mass index $\left(\mathrm{kg} / \mathrm{m}^{2}\right)$ & $0.082(0.009)$ & $0.086(0.008)$ & - & - \\
\hline Triglycerides (mg/dl) & $0.078(0.025)$ & - & - & - \\
\hline $\mathrm{R}^{2}$ & $0.119(<0.001)$ & $0.046(0.001)$ & $0.089(<0.001)$ & $0.072(<0.001)$ \\
\hline
\end{tabular}

\section{Discussion}

The effects of BMI and TG level on BP varied in elderly individuals. In the present study, we determined the prevalence of hypertension and its association with BMI and TG level. Our findings show that the prevalence of hypertension was $36.91 \%(668 / 1810)$ in men, $43.82 \%(797 / 1819)$ in women, and $40.37 \%$ (1465/3629) overall. After stratification by age, the prevalence of hypertension was $39.20 \%$ (481/1227) in men (age $\geq 65$ years), 49.91\% (576/1154) in women (age $\geq 65$ years), and $44.39 \%$ (1057/2381) overall. In a sample collected from the Korea National Health and Nutrition Examination Survey (2007) in the elderly Korean population (age $\geq$ 65 years)[35], the prevalence of hypertension was $62.0 \%$; the prevalence of hypertension in the Japan Gerontological Evaluation Survey[36], conducted in a population aged 65 or older, was $59.54 \%$. The prevalence of hypertension in our study was lower than that observed in the Korean and Japanese studies. Moreover, men with hypertension had a higher prevalence of risk factors, such as age, marital status, eating habits, FPG, LDL-C, TG, eGFR, BMI, and SUA level, than those with normotension. Age, marital status, antilipidemic medication, FPG, TG, eGFR, and SUA level were significantly associated with BP among women. Furthermore, TG levels were positively associated with SBP and DBP in men with $\mathrm{BMI}<24 \mathrm{~kg} / \mathrm{m}^{2}$; however, TG levels were positively associated with both SBP and DBP in women with a BMI < $24 \mathrm{~kg} / \mathrm{m}^{2}$ and also positively associated with DBP in women with a $\mathrm{BMI} \geq 24 \mathrm{~kg} / \mathrm{m}^{2}$.

Studies [37, 38] have explored the association and/or interaction analysis between BMI and SUA level and BP. 
TABLE 12: Multivariate-adjusted relationship between various characteristics and blood pressure status of participants categorized by age in male $(\mathrm{N}=1810)$.

\begin{tabular}{|c|c|c|c|c|}
\hline \multirow[b]{2}{*}{ Variables } & \multicolumn{2}{|c|}{$\begin{array}{c}\text { Age }<68 \text { years } \\
(\mathrm{n}=807)\end{array}$} & \multicolumn{2}{|c|}{$\begin{array}{c}\text { Age } \geq 68 \text { years } \\
(\mathrm{n}=1003)\end{array}$} \\
\hline & $\begin{array}{l}\text { Systolic blood } \\
\text { pressure } \\
\beta(P \text {-value }) \\
\end{array}$ & $\begin{array}{l}\text { Systolic blood } \\
\text { pressure } \\
\beta(P \text {-value }) \\
\end{array}$ & $\begin{array}{l}\text { Systolic blood } \\
\text { pressure } \\
\beta(P \text {-value }) \\
\end{array}$ & $\begin{array}{c}\text { Systolic blood } \\
\text { pressure } \\
\beta(P \text {-value }) \\
\end{array}$ \\
\hline Age & - & - & - & $-0.085(0.015)$ \\
\hline $\begin{array}{l}\text { Education }(0=\text { Illiterate, } 1=\text { Less than } \\
\text { elementary school, } 2=\text { High school, } 3=\text { Above } \\
\text { vocational school) }\end{array}$ & - & - & - & - \\
\hline Marital status $(0=$ Single, $1=$ Married $)$ & $0.078(0.024)$ & $0.1(0.004)$ & $0.074(0.020)$ & - \\
\hline Current residence $(0=$ Rural, $1=$ Urban $)$ & - & - & $0.105(0.002)$ & $0.084(0.014)$ \\
\hline $\begin{array}{l}\text { Smoke }(0=\text { NO, } 1=\text { Former smoke }, 2=\text { Current } \\
\text { smoke })\end{array}$ & - & - & $0.078(0.016)$ & - \\
\hline $\begin{array}{l}\text { Drinking }(0=\text { NO, } 1=\text { Less than once a month, } 2= \\
\text { More than once } a \text { month })\end{array}$ & - & - & - & - \\
\hline $\begin{array}{l}\text { Eating meals }(0=\leq 2 \text { meals per day, } 1=3 \text { meals } \\
\text { per day, } 2=\geq 4 \text { meals per day })\end{array}$ & $-0.070(0.039)$ & $-0.103(0.003)$ & - & $-0.071(0.025)$ \\
\hline Taking activities $(0=$ No, $1=$ Yes $)$ & $-0.077(0.027)$ & - & - & - \\
\hline $\begin{array}{l}\text { Ever been in major accidental injury }(0=\text { No, } 1= \\
\text { Yes })\end{array}$ & - & - & - & - \\
\hline $\begin{array}{l}\text { Having regular physical exercises }(0=\text { No } \\
\text { physical exercise, } 1=\text { Less than regular physical } \\
\text { exercises, } 2=\text { Regular physical exercises })\end{array}$ & - & - & - & - \\
\hline History of $\operatorname{CVD}(0=$ No, $1=$ Yes $)$ & - & - & - & - \\
\hline History of liver diseases $(0=$ No, $1=$ Yes $)$ & - & - & - & - \\
\hline Antilipidemic medication $(0=$ No, $1=$ Yes $)$ & - & - & - & - \\
\hline Anti-diabetic medication $(0=$ No, $1=$ Yes $)$ & - & $-0.078(0.046)$ & - & - \\
\hline Anti-hypertensive therapy $(0=$ No, $1=$ Yes $)$ & $0.09(0.009)$ & $0.090(0.010)$ & $0.111(<0.001)$ & $0.114(<0.001)$ \\
\hline Fasting plasma glucose(mg/dl) & $0.09(0.022)$ & - & - & - \\
\hline LDL Cholesterol (mg/dl) & - & - & - & - \\
\hline HDL Cholesterol (mg/dl) & - & - & - & - \\
\hline $\mathrm{eGFR}\left(\mathrm{ml} / \mathrm{min} / 1.73 \mathrm{~m}^{2}\right)$ & $-0.078(0.040)$ & - & - & - \\
\hline Serum uric acid(mg/dl) & $0.088(0.024)$ & $0.08(0.042)$ & - & - \\
\hline Body mass index $\left(\mathrm{kg} / \mathrm{m}^{2}\right)$ & $0.229(<0.001)$ & $0.213(<0.001)$ & $0.137(<0.001)$ & $0.105(0.003)$ \\
\hline Triglycerides (mg/dl) & $0.024(0.545)$ & $0.03(0.452)$ & $0.096(0.009)$ & $0.063(0.088)$ \\
\hline$\underline{\mathrm{R}^{2}}$ & $0.136(<0.001)$ & $0.115(<0.001)$ & $0.087(<0.001)$ & $0.065(<0.001)$ \\
\hline
\end{tabular}

Lyngdoh et al. [37] reported that adiposity substantially decreased the association between SUA level and BP in young adults, and BP was independently associated with SUA level in women. Kawamoto et al. [38] concluded that BMI changes the association between SUA level and BP status among community-dwelling men. In other studies [39, 40], the association of TG and SUA levels has been persistent after full adjustment in a multiple logistic model, suggesting that TG levels correlate independently with SUA level, with TG levels having the most influence on SUA. However, there were no previous studies on the interactive effect between BMI and TG level on BP.

As our general linear models adjusting for 13 related potential confounders showed, interaction between BMI and TG on BP was not observed. In our investigation of the relationships between BMI or TG and hypertension, we made several observations. First, we found that overweight and obese men and women were more likely to have hypertension. We also observed that women with high TG were more likely to experience hypertension. Thirdly, no association between TG and hypertension was found in men. Lastly, the relative results in relations between BMI or TG and hypertension categorized by age in both men and women are the same as those above. Furthermore, age differences in relations between BMI or TG and hypertension were found. Specifically, individuals with age $\geq 68$ years experienced lower effects of BMI or TG level on BP. Similar evidence has been found in Brazil, in a study based on 287 men and women aged between 18 and 88 years, in which Pimenta [41] found that central obesity and TG level 
TABLE 13: Multivariate-adjusted relationship between various characteristics and blood pressure status of participants categorized by age in female $(\mathrm{N}=1819)$.

\begin{tabular}{|c|c|c|c|c|}
\hline \multirow[b]{2}{*}{ Variables } & \multicolumn{2}{|c|}{$\begin{array}{c}\text { Age }<68 \text { years } \\
(\mathrm{n}=887)\end{array}$} & \multicolumn{2}{|c|}{$\begin{array}{l}\text { Age } \geq 68 \text { years } \\
\quad(n=932)\end{array}$} \\
\hline & $\begin{array}{l}\text { Systolic blood } \\
\text { pressure } \\
\beta(P \text {-value }) \\
\end{array}$ & $\begin{array}{l}\text { Systolic blood } \\
\text { pressure } \\
\beta(P \text {-value }) \\
\end{array}$ & $\begin{array}{l}\text { Systolic blood } \\
\text { pressure } \\
\beta(P \text {-value }) \\
\end{array}$ & $\begin{array}{c}\text { Systolic blood } \\
\text { pressure } \\
\beta(P \text {-value })\end{array}$ \\
\hline Age & - & - & $0.105(0.005)$ & - \\
\hline $\begin{array}{l}\text { Education }(0=\text { Illiterate, } 1=\text { Less than } \\
\text { elementary school, } 2=\text { High school, } 3=\text { Above } \\
\text { vocational school })\end{array}$ & - & - & - & - \\
\hline Marital status $(0=$ Single, $1=$ Married $)$ & $0.071(0.035)$ & - & $0.103(0.003)$ & $0.071(0.043)$ \\
\hline Current residence $(0=$ Rural, $1=$ Urban $)$ & - & - & - & - \\
\hline $\begin{array}{l}\text { Smoke }(0=\mathrm{NO}, 1=\text { Former smoke, } 2=\text { Current } \\
\text { smoke) }\end{array}$ & - & - & - & - \\
\hline $\begin{array}{l}\text { Drinking }(0=\mathrm{NO}, 1=\text { Less than once a } \\
\text { month, } 2=\text { More than once a month })\end{array}$ & - & - & - & - \\
\hline $\begin{array}{l}\text { Eating meals }(0=\leq 2 \text { meals per day, } 1=3 \text { meals } \\
\text { per day, } 2=\geq 4 \text { meals per day) }\end{array}$ & - & $-0.08(0.015)$ & - & - \\
\hline Taking activities $(0=$ No, $1=$ Yes $)$ & - & - & - & - \\
\hline $\begin{array}{l}\text { Ever been in major accidental injury }(0=\text { No, } 1= \\
\text { Yes) }\end{array}$ & - & - & - & - \\
\hline $\begin{array}{l}\text { Having regular physical exercises }(0=\text { No } \\
\text { physical exercise, } 1=\text { Less than regular physical } \\
\text { exercises, } 2=\text { Regular physical exercises })\end{array}$ & - & - & - & - \\
\hline History of CVD ( $0=$ No, $1=$ Yes $)$ & - & - & - & $-0.069(0.048)$ \\
\hline History of liver diseases $(0=$ No, $1=$ Yes $)$ & - & - & - & $-0.076(0.023)$ \\
\hline Antilipidemic medication $(0=$ No, $1=$ Yes $)$ & - & - & - & - \\
\hline Anti-diabetic medication $(0=\mathrm{No}, 1=\mathrm{Yes})$ & $0.105(0.004)$ & - & - & - \\
\hline Anti-hypertensive therapy $(0=$ No,1 $=$ Yes $)$ & $0.085(0.012)$ & - & $0.153(<0.001)$ & $0.089(0.008)$ \\
\hline Fasting plasma glucose (mg/dl) & - & $0.128(<0.001)$ & - & - \\
\hline LDL Cholesterol (mg/dl) & - & - & - & - \\
\hline HDL Cholesterol (mg/dl) & - & - & - & - \\
\hline $\mathrm{eGFR}\left(\mathrm{ml} / \mathrm{min} / 1.73 \mathrm{~m}^{2}\right)$ & - & - & - & - \\
\hline Serum uric acid (mg/dl) & - & - & - & - \\
\hline Body mass index $\left(\mathrm{kg} / \mathrm{m}^{2}\right)$ & $0.11(0.003)$ & $0.23(<0.001)$ & $0.097(0.007)$ & $0.105(0.004)$ \\
\hline Triglycerides (mg/dl) & $0.087(0.031)$ & $0.056(0.159)$ & $0.046(0.270)$ & $0.072(0.090)$ \\
\hline $\mathrm{R}^{2}$ & $0.087(<0.001)$ & $0.110(<0.001)$ & $0.079(<0.001)$ & $0.057(<0.001)$ \\
\hline
\end{tabular}

were independent risk factors for hypertension according to multivariate analysis. However, several studies have reported divergent findings regarding the association between obesity and hypertension. Some studies have suggested an increased risk of hypertension with higher BMI, waist circumference (WC), and waist-to-hip ratio (WHR) [42, 43], whereas other studies found that the association was not significant [4446]. Zhou [47] conducted the first meta-analysis of cohort studies to quantify the relationship between obesity and the incidence of hypertension and found a positive association between the risk of hypertension and BMI, WC, and WHR.
Arabshahi [48] conducted the first meta-analysis of crosssectional studies to investigate the relationship between $\mathrm{BMI}$ or WC and hypertension, and concluded that the risk of hypertension was associated with adiposity. Jayedi [49] conducted a meta-analysis of prospective cohort studies to report the risk estimates of hypertension for abdominal adiposity (BMI, WC, and WHR) and found that the risk of hypertension increased with a somewhat steeper trend with increasing BMI, in comparison with WC and WHR. Such discrepancies between our findings and the null studies may be a result of methodological differences in design, 
TABLE 14: Interaction between body mass index and uric acid on blood pressure status in male and female $(\mathrm{N}=3629)$.

\begin{tabular}{|c|c|c|c|c|}
\hline \multirow[b]{2}{*}{ Characteristics } & \multicolumn{2}{|c|}{$\operatorname{Male}(\mathrm{n}=1810)$} & \multicolumn{2}{|c|}{ Female $(\mathrm{n}=1819)$} \\
\hline & $\begin{array}{c}\text { Systolic blood } \\
\text { pressure } \\
\beta(P \text {-value }) \\
\end{array}$ & $\begin{array}{c}\text { Diastolic blood } \\
\text { pressure } \\
\beta(P \text {-value }) \\
\end{array}$ & $\begin{array}{c}\text { Systolic blood } \\
\text { pressure } \\
\beta(P \text {-value }) \\
\end{array}$ & $\begin{array}{c}\text { Diastolic blood } \\
\text { pressure } \\
\beta(P \text {-value }) \\
\end{array}$ \\
\hline Age(years) & $0.337(0.001)$ & $-0.240(<0.001)$ & $-0.092(0.042)$ & $-0.113(0.013)$ \\
\hline $\begin{array}{l}\text { Education }(0=\text { Illiterate, } 1=\text { Less than } \\
\text { elementary school, } 2=\text { High school, } 3=\text { Above } \\
\text { vocational school) }\end{array}$ & - & - & - & $-1.119(0.019)$ \\
\hline Marital status $(0=$ Single, $1=$ Married $)$ & $-5.660(0.001)$ & $-2.494(0.004)$ & $-1.503(0.024)$ & $-1.453(0.029)$ \\
\hline Current residence $(0=$ Rural, $1=$ Urban $)$ & $3.574(0.003)$ & - & - & - \\
\hline $\begin{array}{l}\text { Smoke }(0=\text { NO, } 1=\text { Former smoke }, 2=\text { Current } \\
\text { smoke })\end{array}$ & $1.864(0.005)$ & - & - & - \\
\hline $\begin{array}{l}\text { Eating meals }(0=\leq 2 \text { meals per day, } 1=3 \text { meals } \\
\text { per day, } 2=\geq 4 \text { meals per day })\end{array}$ & $2.978(0.045)$ & $2.483(0.002)$ & - & $2.193(0.003)$ \\
\hline Taking activities $(0=$ No, $1=$ Yes $)$ & - & - & - & - \\
\hline Anti-diabetic medication $(0=$ No, $1=$ Yes $)$ & - & - & - & - \\
\hline Anti-hypertensive therapy $(0=$ No, $1=$ Yes $)$ & $10.228(<0.001)$ & $5.745(<0.001)$ & $3.292(0.003)$ & - \\
\hline Fasting plasma glucose $(\mathrm{mg} / \mathrm{dl})$ & $0.039(0.011)$ & - & - & - \\
\hline LDL Cholesterol (mg/dl) & $0.049(0.004)$ & - & - & - \\
\hline $\mathrm{eGFR}\left(\mathrm{ml} / \mathrm{min} / 1.73 \mathrm{~m}^{2}\right)$ & $-0.123(0.003)$ & - & - & - \\
\hline Serum uric $\operatorname{acid}(\mathrm{mg} / \mathrm{dl})$ & $0.948(0.047)$ & - & - & - \\
\hline Body mass index & $-8.004(0.002)$ & $-4.125(0.002)$ & $-3.668(<0.001)$ & $-4.102(<0.001)$ \\
\hline Triglycerides & $-3.962(0.076)$ & $-1.482(0.203)$ & $-2.140(0.017)$ & $-2.319(0.009)$ \\
\hline Body mass index $*$ Triglycerides & $0.572(0.845)$ & $-0.373(0.810)$ & $0.122(0.923)$ & $0.272(0.828)$ \\
\hline
\end{tabular}

measurement of obesity, and populations. Moreover, TG level has been associated with BP since TG level itself can cause endothelial dysfunction [24, 25], arterial stiffness [26], and the loss of vasomotor reactivity [27]. Such pathophysiology induced by increased TG and FPG levels and low HDL-C level may be greater than that of SUA. To explore the extent of the effects of TG level on blood pressure, we controlled for SUA and the related confounders, such as LDL-C and HDL$\mathrm{C}$ [38]. The findings showed that TG level and hypertension were observed in women, but no independent effect was observed in men. Sanchez-Inigo [50] conducted a cohort study to identify the association of TG with the incidence of hypertension in Spain and found that the incidence of hypertension was associated with TG level in both men and women independent of adiposity. Tohidi [51] found that high TG independently predicted incidence of hypertension in Middle Eastern women.

The mechanisms that lead to hypertension in participants with high BMI or TG levels have not been completely understood. Current studies may provide insight into the pathogenic mechanisms of BMI/TG that induce hypertension. The present study suggests that TG level may play an important role in hypertension in women. We speculate that sex-specific factors may also play an important role. TG levels are higher in women than in men, which partially explains the underlying mechanism that accounts for sex difference based on hormone levels. Additionally, body fat, sex steroids, and their interaction in elderly participants may also be associated with hypertension. As an important sex hormone, estrogen may also affect BP. Elderly women have lower concentrations of estrogen, which may affect the level of TG and result in a smaller protective effect.

\section{Strengths and Limitations of the Study}

There are several limitations of our study. First, the association and interaction of BMI and serum TG on blood pressure become seriously more complex; we only consider the confounders as possible as we can, but there are some unknown factors. Secondly, the relationship should be studied prospectively. Our study investigated BP in the elderly participant through a cross-sectional study. Follow-up study was relatively short to comprehensively observe changes in the next step. Last, more research is needed to observe the result. Several strengths could be found in our study. Firstly, the study was based on a nationwide survey. Secondly, we conducted the analyses according to gender.

\section{Conclusions}

An interactive effect of BMI and TG level on BP was not observed in either men or women; however, independent 
TABLE 15: Interaction between body mass index and uric acid on blood pressure status of participants categorized by age in male and female $(\mathrm{N}=3629)$.

\begin{tabular}{|c|c|c|c|c|}
\hline \multirow{2}{*}{$\begin{array}{l}\text { Characteristics } \\
\text { Male }\end{array}$} & \multicolumn{2}{|c|}{$\begin{array}{l}\text { Age }<68 \text { years } \\
(\mathrm{n}=807)\end{array}$} & \multicolumn{2}{|c|}{$\begin{array}{l}\text { Age } \geq 68 \text { years } \\
(\mathrm{n}=1003)\end{array}$} \\
\hline & $\begin{array}{c}\text { Systolic blood } \\
\text { pressure } \\
\beta(P \text {-value })\end{array}$ & $\begin{array}{l}\text { Diastolic blood } \\
\text { pressure } \\
\beta(P \text {-value }) \\
\end{array}$ & $\begin{array}{c}\text { Systolic blood } \\
\text { pressure } \\
\beta(P \text {-value }) \\
\end{array}$ & $\begin{array}{c}\text { Diastolic blood } \\
\text { pressure } \\
\beta(P \text {-value })\end{array}$ \\
\hline Marital status $(0=$ Single, $1=$ Married $)$ & $-6.913(0.009)$ & $-5.237(0.001)$ & $-5.876(0.007)$ & \\
\hline Current residence $(0=$ Rural, $1=$ Urban $)$ & & & $5.232(0.004)$ & $2.164(0.015)$ \\
\hline $\begin{array}{l}\text { Smoke }(0=\mathrm{NO}, 1=\text { Former smoke }, 2=\text { Current } \\
\text { smoke) }\end{array}$ & & & $1.917(0.05)$ & \\
\hline $\begin{array}{l}\text { Eating meals }(0=\leq 2 \text { meals per day, } 1=3 \text { meals } \\
\text { per day, } 2=\geq 4 \text { meals per day) }\end{array}$ & $3.746(0.058)$ & $3.227(0.006)$ & & $2.239(0.036)$ \\
\hline Taking activities $(0=$ No, $1=$ Yes $)$ & $-2.747(0.057)$ & & & \\
\hline Antilipidemic medication $(0=$ No, $1=$ Yes $)$ & & $-1.651(0.423)$ & & \\
\hline Anti-hypertensive therapy $(0=$ No, $1=$ Yes $)$ & $9.032(0.002)$ & $5.343(0.002)$ & $11.982(<0.001)$ & $6.384(<0.001)$ \\
\hline Fasting plasma glucose (mg/dl) & $0.040(0.030)$ & & & \\
\hline $\mathrm{eGFR}\left(\mathrm{ml} / \mathrm{min} / 1.73 \mathrm{~m}^{2}\right)$ & $-0.122(0.024)$ & & & \\
\hline Serum uric acid(mg/dl) & $1.454(0.022)$ & $0.957(0.006)$ & & \\
\hline Body mass index & $-8.409(0.006)$ & $-4.837(0.008)$ & $-8.347(0.048)$ & $-3.042(0.142)$ \\
\hline Triglycerides & $-0.135(0.958)$ & $-0.587(0.695)$ & $-7.960(0.035)$ & $-1.478(0.427)$ \\
\hline Body mass index $*$ Triglycerides & $-1.075(0.760)$ & $-0.095(0.964)$ & $2.138(0.654)$ & $-0.931(0.691)$ \\
\hline \multirow[t]{2}{*}{ Female } & \multicolumn{2}{|c|}{$\begin{array}{l}\text { Age }<68 \text { years } \\
\quad(\mathrm{n}=887)\end{array}$} & \multicolumn{2}{|c|}{$\begin{array}{l}\text { Age } \geq 68 \text { years } \\
\quad(\mathrm{n}=932)\end{array}$} \\
\hline & $\begin{array}{l}\text { Systolic blood } \\
\text { pressure } \\
\beta(P \text {-value })\end{array}$ & $\begin{array}{l}\text { Diastolic blood } \\
\text { pressure } \\
\beta(P \text {-value })\end{array}$ & $\begin{array}{l}\text { Systolic blood } \\
\text { pressure } \\
\beta(P \text {-value })\end{array}$ & $\begin{array}{l}\text { Diastolic blood } \\
\text { pressure } \\
\beta(P \text {-value })\end{array}$ \\
\hline Age & - & - & $0.626(0.001)$ & - \\
\hline $\begin{array}{l}\text { Education }(0=\text { Illiterate, } 1=\text { Less than } \\
\text { elementary school, } 2=\text { High school, } 3=\text { Above } \\
\text { vocational school })\end{array}$ & - & - & - & - \\
\hline Marital status ( $0=$ Single, $1=$ Married $)$ & $5.866(0.012)$ & - & $5.481(0.007)$ & $7.504(<0.001)$ \\
\hline $\begin{array}{l}\text { Eating meals }(0=\leq 2 \text { meals per day, } 1=3 \text { meals } \\
\text { per day, } 2=\geq 4 \text { meals per day) }\end{array}$ & - & $2.821(0.009)$ & - & - \\
\hline History of CVD (0= No,1= Yes) & - & - & - & $-3.837(0.123)$ \\
\hline Antilipidemic medication $(0=$ No, $1=$ Yes $)$ & - & - & - & - \\
\hline Anti-diabetic medication $(0=$ No, $1=$ Yes $)$ & $11.822(0.002)$ & - & - & - \\
\hline Anti-hypertensive therapy $(0=$ No, $1=$ Yes $)$ & $8.946(0.006)$ & - & $17.459(<0.001)$ & $17.254(<0.001)$ \\
\hline Fasting plasma glucose (mg/dl) & - & $0.035(<0.001)$ & - & \\
\hline Body mass index & $-5.696(0.062)$ & $-6.621(<0.001)$ & $-4.726(0.187)$ & $-5.152(0.155)$ \\
\hline Triglycerides & $-4.716(0.063)$ & $-2.716(0.024)$ & $-1.573(0.622)$ & $-2.147(0.507)$ \\
\hline Body mass index $*$ Triglycerides & $-1.345(0.718)$ & $2.410(0.170)$ & $-4.192(0.334)$ & $-3.386(0.439)$ \\
\hline
\end{tabular}

effects of BMI on BP were observed in both men and women, and an association between TG level and hypertension was observed in women.

\section{Abbreviations}

CHARLS: China Health and Retirement Longitudinal Study

BMI: $\quad$ Body mass index

BP: $\quad$ Blood pressure

DBP: Diastolic blood pressure
SBP: Systolic blood pressure

SUA: Serum uric acid

CVD: Cardiovascular disease

M: Mean

eGFR: Estimated glomerular filtration rate

CDC: Centers for Disease Control and Prevention

Scr: Serum creatinine

Scys: Serum cystatin C

SD: $\quad$ Standard deviation

LDL: Low-density lipoprotein

HDL: High-density lipoprotein 


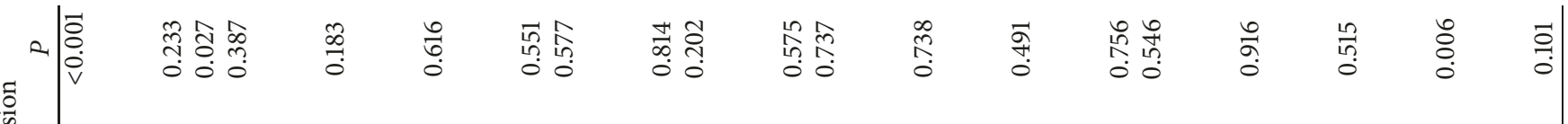

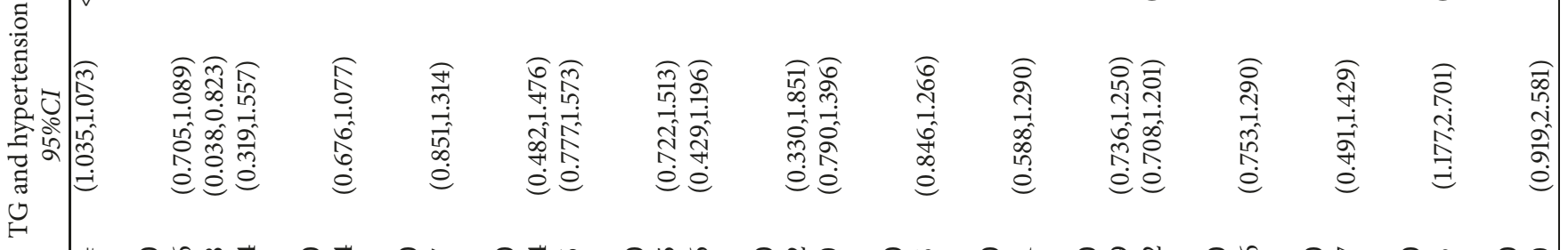
of 萑 $\frac{2}{\tilde{\Sigma}}$

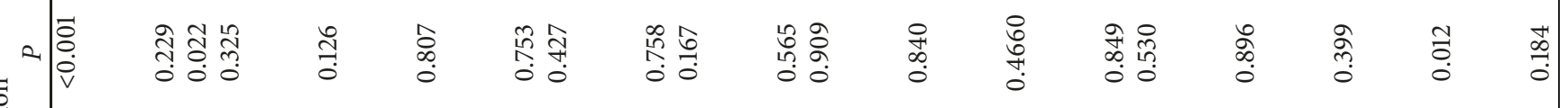

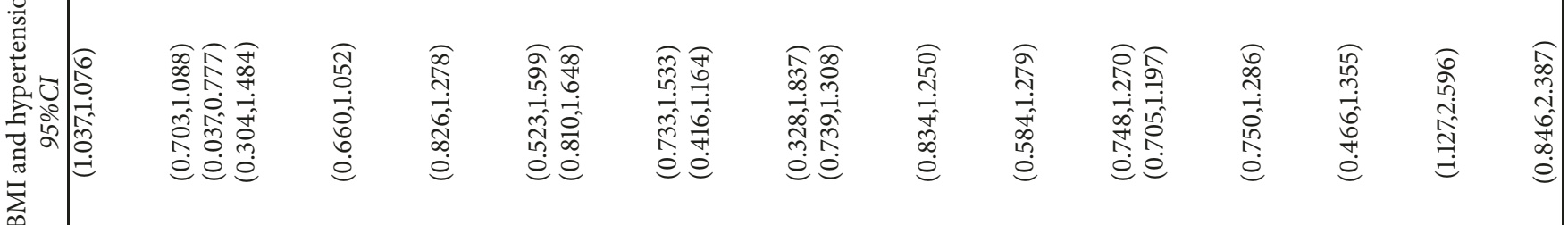

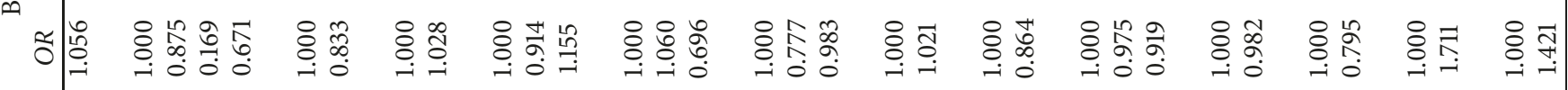

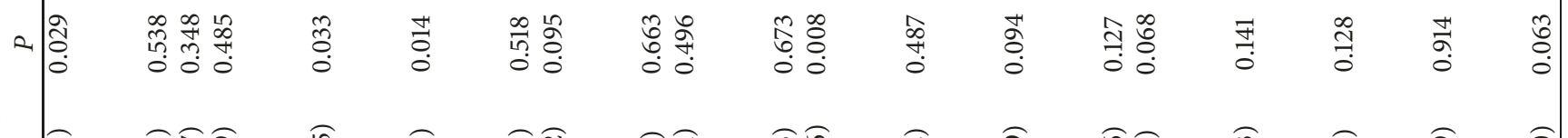

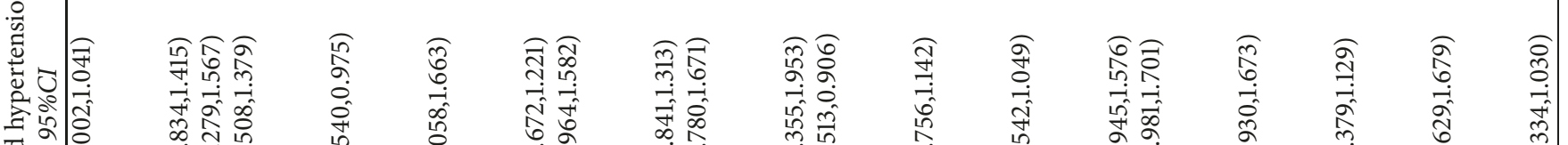

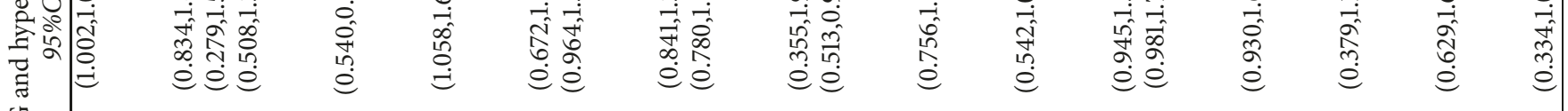
D

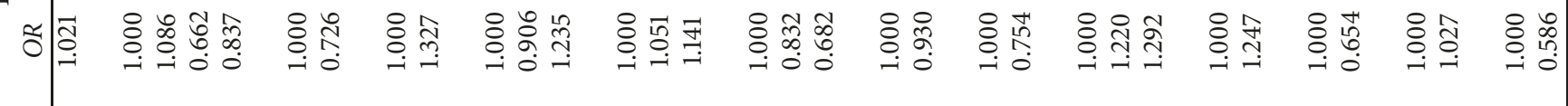
这

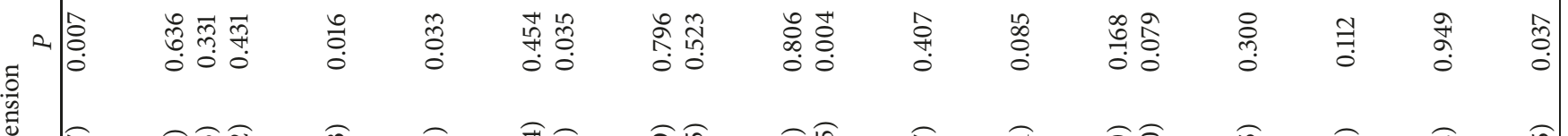

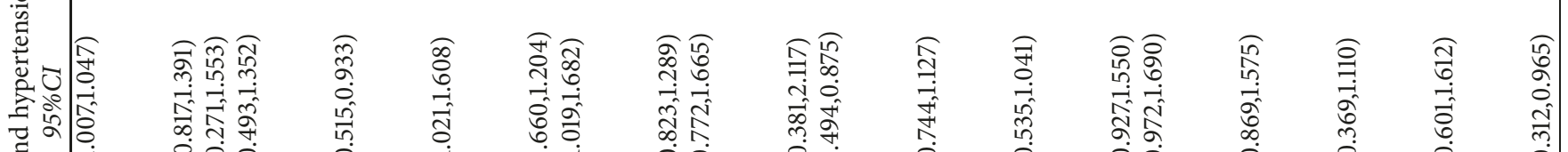

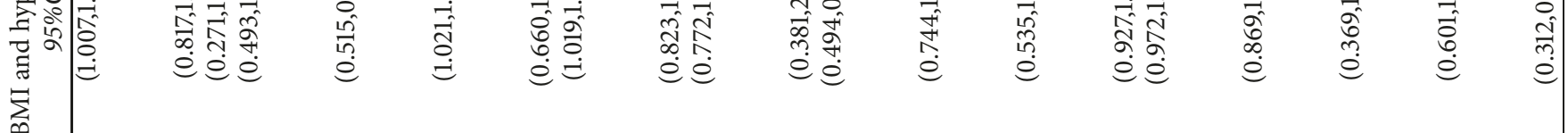
\& |

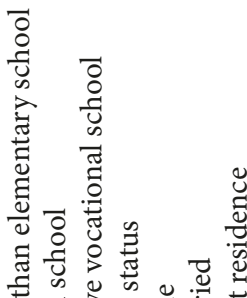

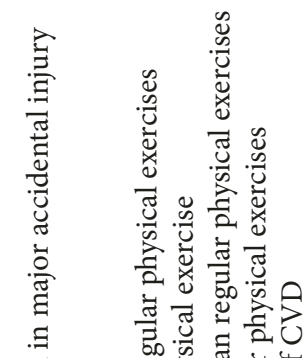

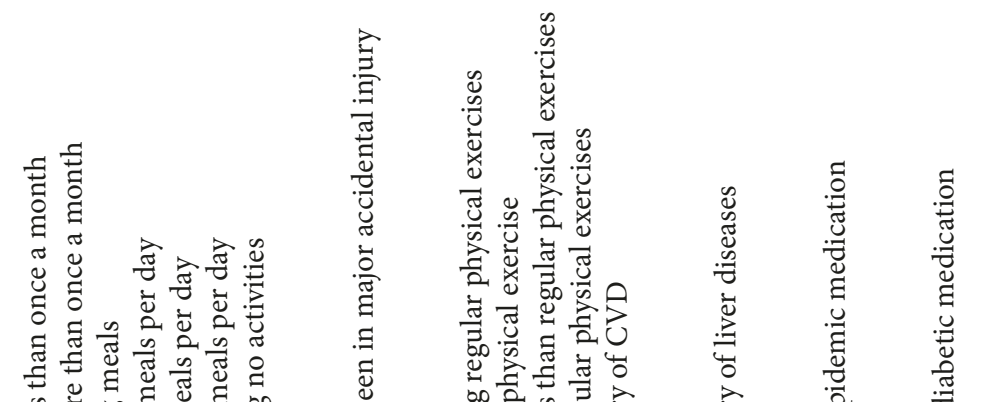

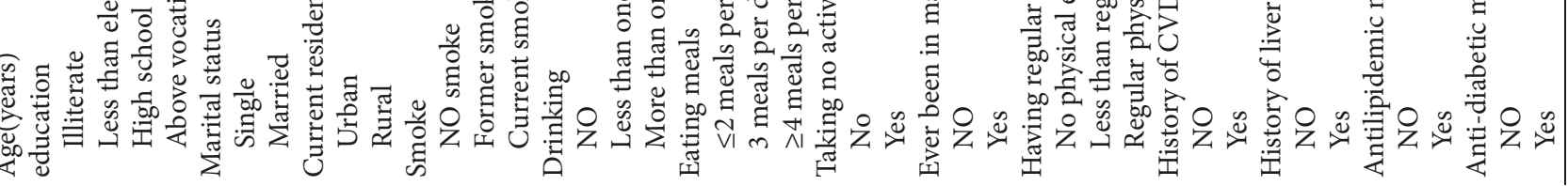




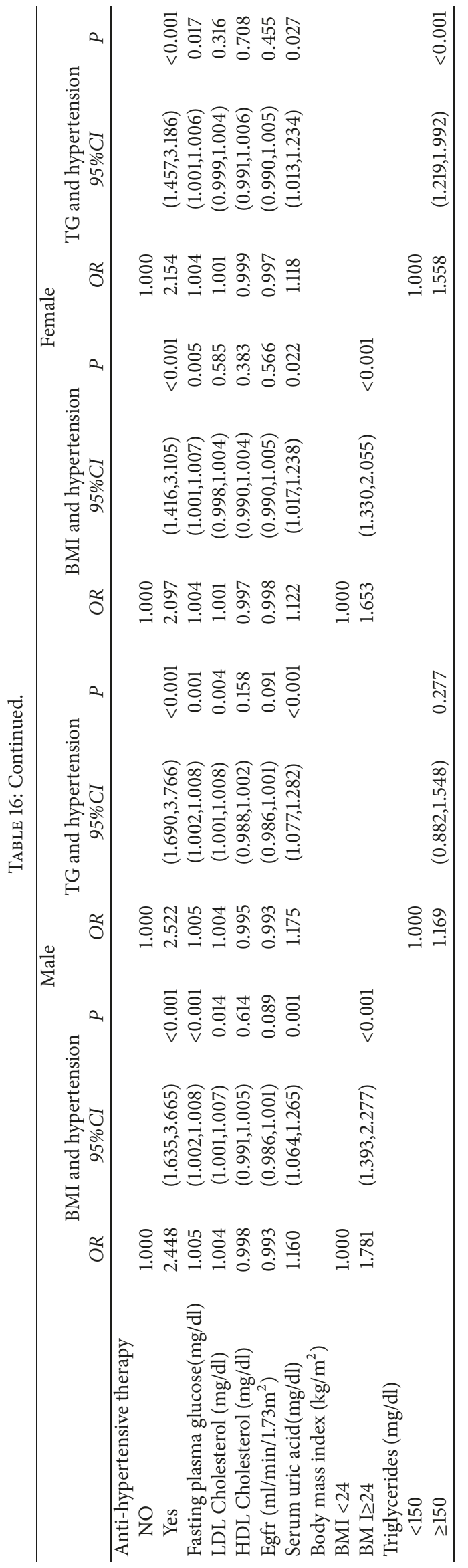




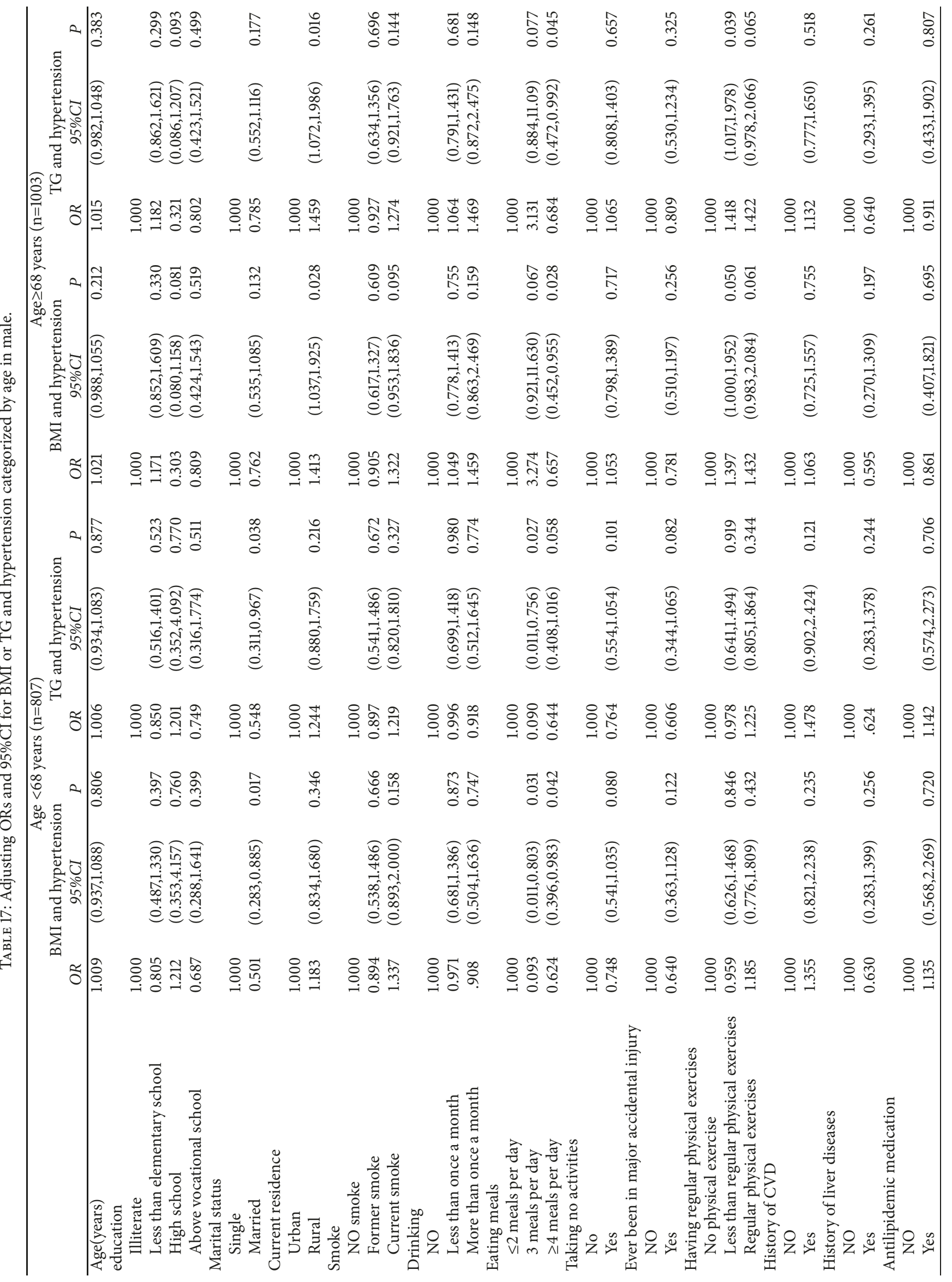




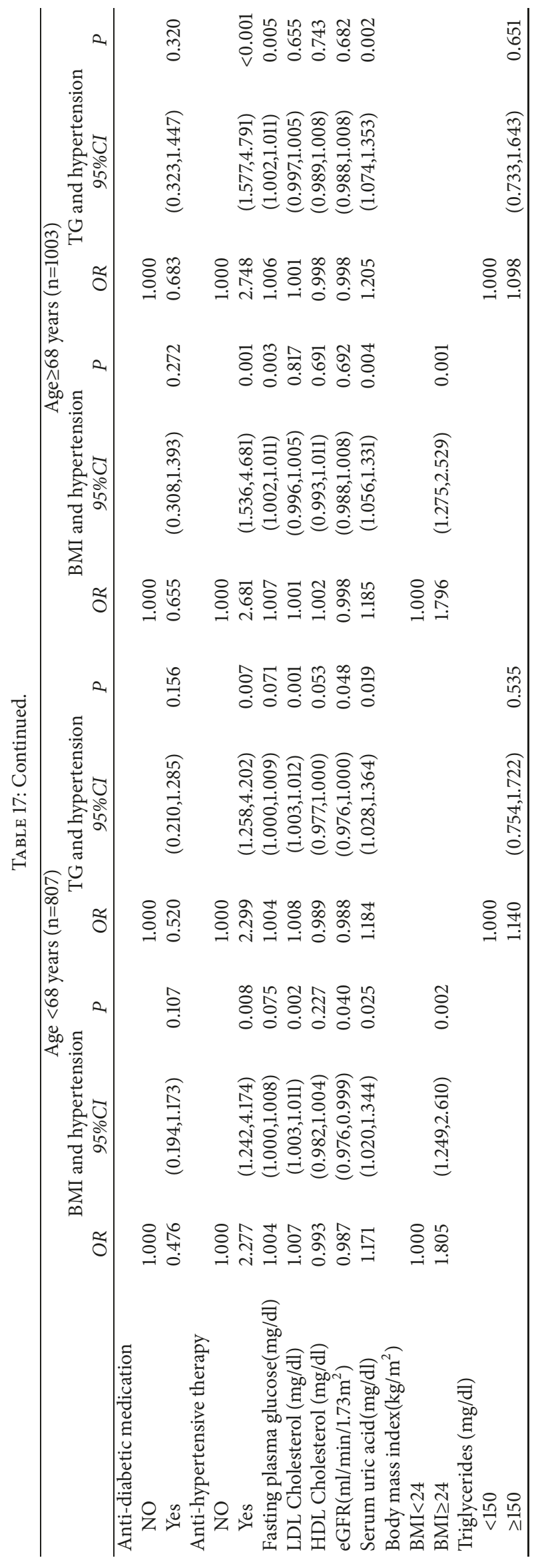




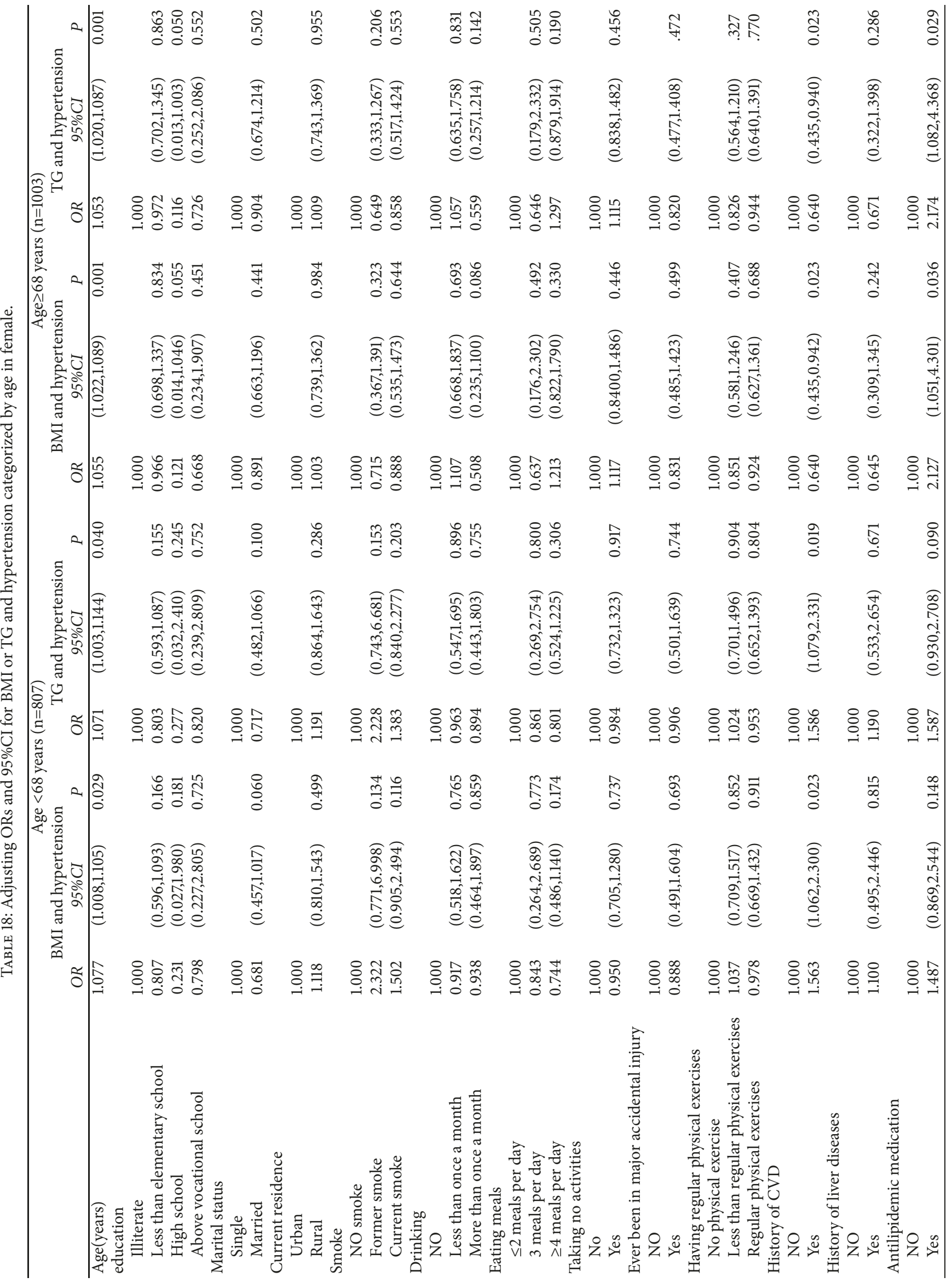




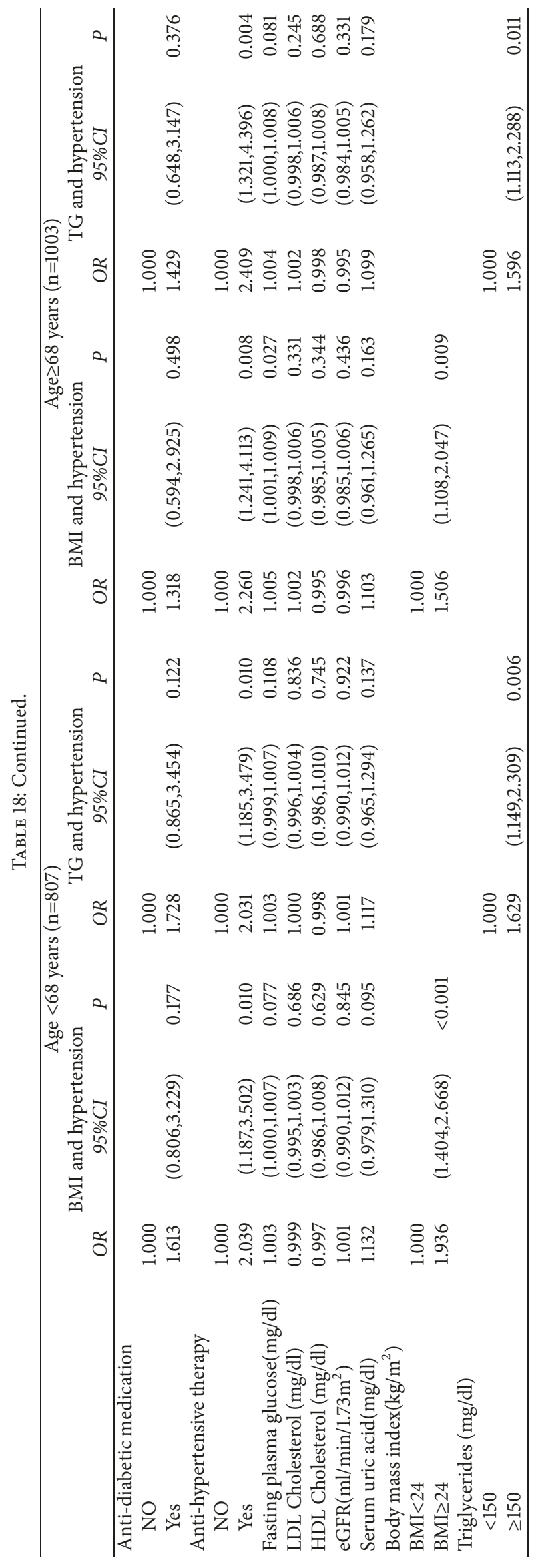


NIA: National intelligence agency

FPG: Fasting plasma glucose

TG: Triglycerides

WC: Waist circumference

WHR: Waist to hip ratio.

\section{Data Availability}

Data sharing statement Extra data can be accessed via http://charls.pku.edu.cn/zh-CN.

\section{Ethical Approval}

The study is publicly available at http://charls.pku.edu.cn/ zh-CN with no direct contact with the individual participants.

\section{Consent}

The patient's consent was obtained.

\section{Disclosure}

The funding body was not involved in the design of the study, data collection, analysis, and interpretation, or in writing the manuscript.

\section{Conflicts of Interest}

The authors declare that they have no conflicts of interest.

\section{Authors' Contributions}

Lin Zhang conceived and drafted the manuscript. Jin-long Li, Li-li Zhang, Lei-lei Guo, Hong Li, and Dan Li helped revise the manuscript. All authors have read and approved the manuscript.

\section{Acknowledgments}

The authors are grateful to the participants and members of the CHARLS. CHARLS was supported by the NIA. Lin Zhang gives thanks to his wife (Haiyang Liu) who stands behind him for supporting him in the past year during writing the paper.

\section{References}

[1] Y. Zhao, H. Yan, R. J. Marshall et al., "Trends in population blood pressure and prevalence, awareness, treatment, and control of hypertension among middle-aged and older adults in a rural area of Northwest China from 1982 to 2010," PLoS ONE, vol. 8, no. 4, p. e61779, 2013.

[2] Y. Wu, R. Huxley, L. Li et al., "Prevalence, awareness, treatment, and control of hypertension in China data from the China National Nutrition and Health Survey 2002," Circulation, vol. 118, no. 25, pp. 2679-2686, 2008.
[3] X. Liu, W. Gu, Z. Li, H. Lei, G. Li, and W. Huang, "Hypertension prevalence, awareness, treatment, control, and associated factors in Southwest China: An update," Journal of Hypertension, vol. 35, no. 3, pp. 637-644, 2017.

[4] L. Wu, Y. He, B. Jiang et al., "Trends in prevalence, awareness, treatment and control of hypertension during 2001-2010 in an urban elderly population of China," PLoS ONE, vol. 10, no. 8, p. e0132814, 2015.

[5] S. Wang, R. Chen, Q. Liu, Z. Shu, S. Zhan, and L. Li, "Prevalence, awareness and treatment of chronic kidney disease among middle-aged and elderly: The China Health and Retirement Longitudinal Study," Nephrology, vol. 20, no. 7, pp. 474-484, 2015.

[6] R. J. Glynn, G. J. L'Italien, H. D. Sesso, E. A. Jackson, and J. E. Buring, "Development of predictive models for long-term cardiovascular risk associated with systolic and diastolic blood pressure," Hypertension, vol. 39, no. 1, pp. 105-110, 2002.

[7] D. Yu, J. Huang, D. Hu et al., "Association between prehypertension and clustering of cardiovascular disease risk factors among chinese adults," Journal of Cardiovascular Pharmacology, vol. 53, no. 5, pp. 388-400, 2009.

[8] D. Gu, J. Chen, X. Wu et al., "Prehypertension and risk of cardiovascular disease in Chinese adults," Journal of Hypertension, vol. 27, no. 4, pp. 721-729, 2009.

[9] R. H. Fagard, "Predicting risk of fatal cardiovascular disease and sudden death in hypertension," Journal of Hypertension, vol. 35, no. 11, pp. 2165-2167, 2017.

[10] J. E. James, "Hypertension control and cardiovascular disease," The Lancet, vol. 389, no. 10065, p. 154, 2017.

[11] D. T. Lackland and M. A. Weber, "Global burden of cardiovascular disease and stroke: hypertension at the core," Canadian Journal of Cardiology, vol. 31, no. 5, pp. 569-571, 2015.

[12] L. Cloutier, D. Morris, J. Bruneau, D. McLean, and N. Campbell, "World Health Organization celebrates World Health Day, April 7,2013-focusing on hypertension," Canadian Journal of Cardiovascular Nursing, vol. 23, no. 2, pp. 9-11, 2013.

[13] Z. Gui, Y. Zhu, L. Cai et al., "Sugar-sweetened beverage consumption and risks of obesity and hypertension in chinese children and adolescents: a national cross-sectional analysis," Nutrients, vol. 9, no. 12, p. 1302, 2017.

[14] M.-S. Lee, C.-H. Chang, R.-Y. Lin, and M.-S. Lai, "Risks of hypertension associated with cyclosporine, nonsteroidal antiinflammatory drugs, and systemic glucocorticoids in patients with psoriasis: A nationwide population-based nested casecontrol study in Taiwan," Pharmacoepidemiology and Drug Safety, vol. 25, no. 2, pp. 133-140, 2016.

[15] W. B. Kannel, "Hypertension: reflections on risks and prognostication," Medical Clinics of North America, vol. 93, no. 3, pp. 541-558, 2009.

[16] L. Beilin, "Alcohol and hypertension: Balancing the risks and benefits," Journal of Hypertension, vol. 23, no. 11, pp. 1953-1955, 2005.

[17] A. Cartier, J. L. Malo, D. Gautrin, L. P. Boulet, and H. Boisjoly, "Glucocorticoid use and risks of ocular hypertension and glaucoma," Journal of the American Medical Association, vol. 277, no. 24, pp. 1929-1930, 1997.

[18] S. A. Isezuo, A. A. Sabir, A. E. Ohwovorilole, and O. A. Fasanmade, "Prevalence, associated factors and relationship between prehypertension and hypertension: a study of two ethnic African populations in Northern Nigeria," Journal of Human Hypertension, vol. 25, no. 4, pp. 224-230, 2011. 
[19] R. Kawamoto, K. Kohara, Y. Tabara, M. Abe, T. Kusunoki, and T. Miki, "Insulin resistance and prevalence of prehypertension and hypertension among community-dwelling persons," Journal of Atherosclerosis and Thrombosis, vol. 17, no. 2, pp. 148-155, 2010.

[20] R. Kawamoto, Y. Tabara, K. Kohara, T. Miki, M. Abe, and T. Kusunoki, "Increased high-density lipoprotein cholesterol is associated with a high prevalence of pre-hypertension and hypertension in community-dwelling persons," Endocrine Journal, vol. 42, no. 2, pp. 321-328, 2012.

[21] J. Zicha, J. Kuneš, and M.-A. Devynck, "Abnormalities of membrane function and lipid metabolism in hypertension: A review," American Journal of Hypertension, vol. 12, no. 3, pp. 315331, 1999.

[22] F. Teng, R. Zhu, C. Zou et al., "Interaction between serum uric acid and triglycerides in relation to blood pressure," Journal of Human Hypertension, vol. 25, no. 11, pp. 686-691, 2011.

[23] P. J. Brennan, J. M. Simpson, R. B. Blacket, and C. A. McGilchrist, "The effects of body weight on serum cholesterol, serum triglycerides, serum urate and systolic blood pressure," Australian and New Zealand Journal of Medicine, vol. 10, no. 1, pp. 15-20, 1980.

[24] M. F. McCarty, "Does postprandial storage of triglycerides in endothelial cells contribute to the endothelial dysfunction associated with insulin resistance and fatty diets?" Medical Hypotheses, vol. 61, no. 2, pp. 167-172, 2003.

[25] A. Jagla and J. Schrezenmeir, "Postprandial triglycerides and endothelial function," Experimental and Clinical Endocrinology \& Diabetes, vol. 109, no. 4, pp. S533-S547, 2001.

[26] W.-M. Yao, H.-F. Zhang, Z.-Y. Zhu et al., "Genetically elevated levels of circulating triglycerides and brachial-ankle pulse wave velocity in a Chinese population," Journal of Human Hypertension, vol. 27, no. 4, pp. 265-270, 2013.

[27] P. Lundman, M. Eriksson, K. Schenck-Gustafsson, F. Karpe, and P. Tornvall, "Transient triglyceridemia decreases vascular reactivity in young, healthy men without risk factors for coronary heart disease," Circulation, vol. 96, no. 10, pp. 32663268, 1997.

[28] C. Li, T. Liu, W. Sun, L. Wu, and Z.-Y. Zou, "Prevalence and risk factors of arthritis in a middle-aged and older Chinese population: The China health and retirement longitudinal study," Rheumatology, vol. 54, no. 4, pp. 697-706, 2014.

[29] B. Ríos-González, B. Ibarra-Cortés, G. Ramírez-López, J. Sánchez-Corona, and M. T. Magaña-Torres, "Association of Polymorphisms of Genes Involved in Lipid Metabolism with Blood Pressure and Lipid Values in Mexican Hypertensive Individuals," Disease Markers, vol. 2014, Article ID 150358, 9 pages, 2014.

[30] L. Huo, L. Ji, W. Deng et al., "Age distribution and metabolic disorders in people with Type 1 diabetes in Beijing and Shantou, China: a cross-sectional study," Diabetic Medicine, vol. 35, no. 6, pp. 721-728, 2018.

[31] Y. Zhu, X. Ye, B. Zhu et al., "Comparisons between the 2012 new CKD-EPI (Chronic Kidney Disease Epidemiology Collaboration) equations and other four approved equations," PLoS ONE, vol. 9, no. 1, Article ID e84688, 2014.

[32] L. Zhang, K. Liu, H. Li et al., "Relationship between body mass index and depressive symptoms: the "fat and jolly" hypothesis for the middle-aged and elderly in China," BMC Public Health, vol. 16, no. 1, 2016.

[33] H.-H. Chang and S. T. Yen, "Association between obesity and depression: Evidence from a longitudinal sample of the elderly in Taiwan," Aging \& Mental Health, vol. 16, no. 2, pp. 173-180, 2012.

[34] B.-F. Zhou, "Effect of body mass index on all-cause mortality and incidence of cardiovascular diseases-report for metaanalysis of prospective studies open optimal cut-off points of body mass index in Chinese adults," Biomedical and environmental sciences : BES, vol. 15, no. 3, pp. 245-252, 2002.

[35] M.-G. Kang, S.-W. Kim, S.-J. Yoon, J.-Y. Choi, K.-I. Kim, and C.H. Kim, "Association between frailty and hypertension prevalence, treatment, and control in the elderly Korean population," Scientific Reports, vol. 7, no. 1, 2017.

[36] A. Yazawa, Y. Inoue, T. Fujiwara et al., "Association between social participation and hypertension among older people in Japan: The JAGES Study," Hypertension Research, vol. 39, no. 11, pp. 818-824, 2016.

[37] T. Lyngdoh, B. Viswanathan, G. J. Myers, M. Bochud, and P. Bovet, "Impact of different adiposity measures on the relation between serum uric acid and blood pressure in young adults," Journal of Human Hypertension, vol. 26, no. 11, pp. 677-683, 2012.

[38] R. Kawamoto, D. Ninomiya, K. Senzaki, and T. Kumagi, "Interaction between body mass index and serum uric acid in relation to blood pressure in community-dwelling Japanese men," Clinical Hypertension, vol. 24, no. 1, 2018.

[39] S. Nejatinamini, A. Ataie-Jafari, M. Qorbani et al., "Association between serum uric acid level and metabolic syndrome components," Journal of Diabetes and Metabolic Disorders, vol. 14, no. 1, p. 70, 2015.

[40] S.-D. Lin, D.-H. Tsai, and S.-R. Hsu, "Association between serum uric acid level and components of the metabolic syndrome," Journal of the Chinese Medical Association, vol. 69, no. 11, pp. 512-516, 2006.

[41] A. M. Pimenta, G. Kac, A. Gazzinelli, R. Corrêa-Oliveira, and G. Velásquez-Meléndez, "Associação entre obesidade central, triglicerídeos e hipertensão arterial em uma área rural do Brasil," Arquivos Brasileiros de Cardiologia, vol. 90, no. 6, pp. 386-392, 2008.

[42] L. R. Grootveld, I. G. M. Van Valkengoed, R. J. G. Peters et al., "The role of body weight, fat distribution and weight change in ethnic differences in the 9-year incidence of hypertension," Journal of Hypertension, vol. 32, no. 5, pp. 990-997, 2014.

[43] F. D. Fuchs, M. Gus, L. B. Moreira et al., "Anthropometrie indices and the incidence of hypertension: a comparative analysis," Obesity Research, vol. 13, no. 9, pp. 1515-1517, 2005.

[44] D. Moliner-Urdiales, E. G. Artero, X. Sui, V. España-Romero, D. C. Lee, and S. N. Blair, "Body adiposity index and incident hypertension: The Aerobics Center Longitudinal Study," Nutrition, Metabolism \& Cardiovascular Diseases, vol. 24, no. 9, pp. 969-975, 2013.

[45] C.-L. Chei, H. Iso, K. Yamagishi et al., "Body fat distribution and the risk of hypertension and diabetes among Japanese men and women," Hypertension Research, vol. 31, no. 5, pp. 851-857, 2008.

[46] P. T. Williams, K. Hoffman, and I. La, "Weight-related increases in hypertension, hypercholesterolemia, and diabetes risk in normal weight male and female runners," Arteriosclerosis, Thrombosis, and Vascular Biology, vol. 27, no. 8, pp. 1811-1819, 2007.

[47] W. Zhou, Y. Shi, Y. Li et al., "Body mass index, abdominal fatness, and hypertension incidence: a dose-response metaanalysis of prospective studies," Journal of Human Hypertension, vol. 32, no. 5, pp. 321-333, 2018. 
[48] S. Arabshahi, D. Busingye, A. K. Subasinghe, R. G. Evans, M. A. Riddell, and A. G. Thrift, "Adiposity has a greater impact on hypertension in lean than not-lean populations: A systematic review and meta-analysis," European Journal of Epidemiology, vol. 29, no. 5, pp. 311-324, 2014.

[49] A. Jayedi, A. Rashidy-Pour, M. Khorshidi, and S. Shab-Bidar, "Body mass index, abdominal adiposity, weight gain and risk of developing hypertension: a systematic review and doseresponse meta-analysis of more than 2.3 million participants," Obesity Reviews, vol. 19, no. 5, pp. 654-667, 2018.

[50] L. Sánchez-Íñigo, D. Navarro-González, J. Pastrana-Delgado, A. Fernández-Montero, and J. A. Martínez, "Association of triglycerides and new lipid markers with the incidence of hypertension in a Spanish cohort," Journal of Hypertension, vol. 34, no. 7, pp. 1257-1265, 2016.

[51] M. Tohidi, M. Hatami, F. Hadaegh, and F. Azizi, “Triglycerides and triglycerides to high-density lipoprotein cholesterol ratio are strong predictors of incident hypertension in Middle Eastern women," Journal of Human Hypertension, vol. 26, no. 9, pp. 525-532, 2012. 


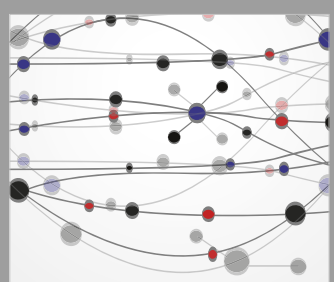

The Scientific World Journal
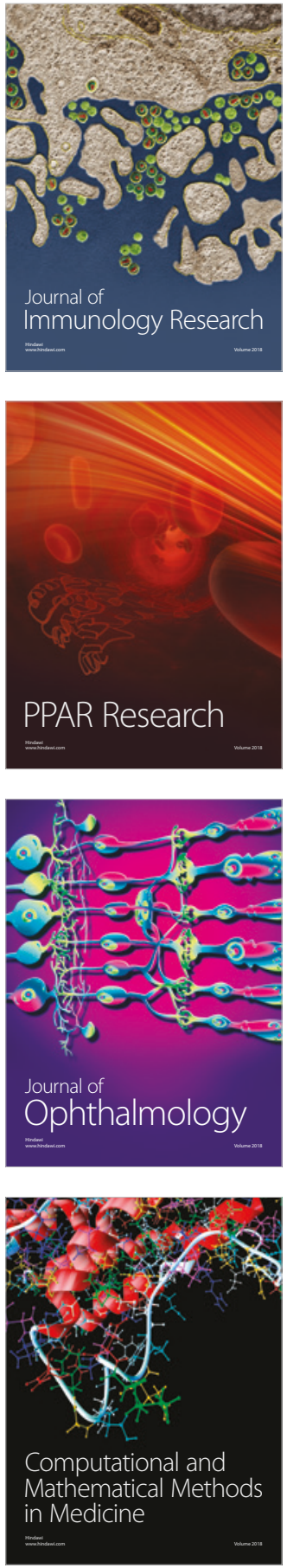

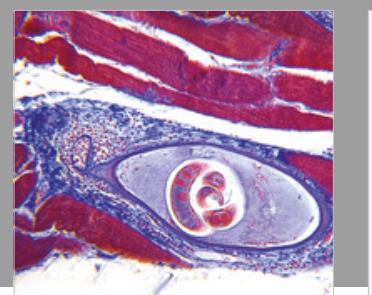

Gastroenterology Research and Practice

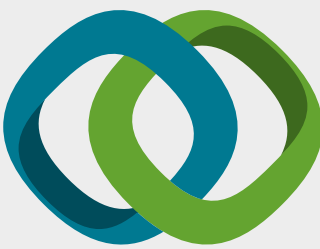

\section{Hindawi}

Submit your manuscripts at

www.hindawi.com
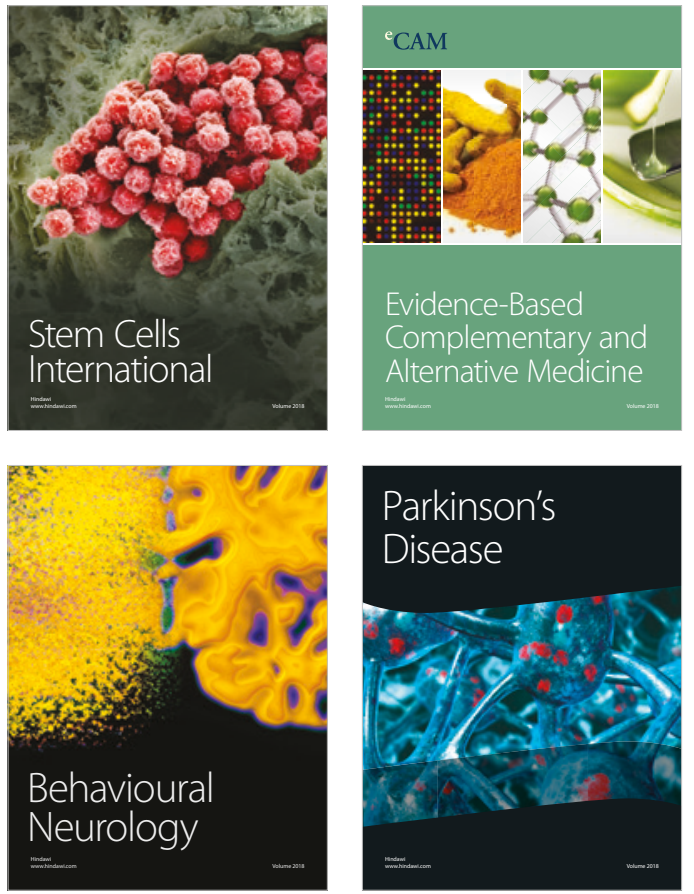

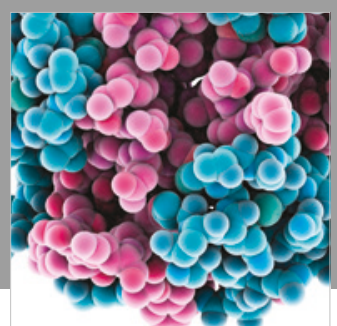

ournal of

Diabetes Research

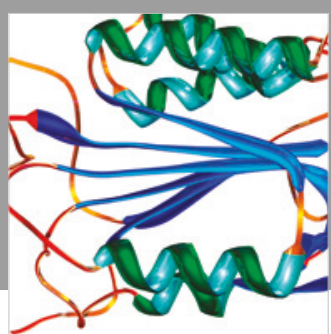

Disease Markers
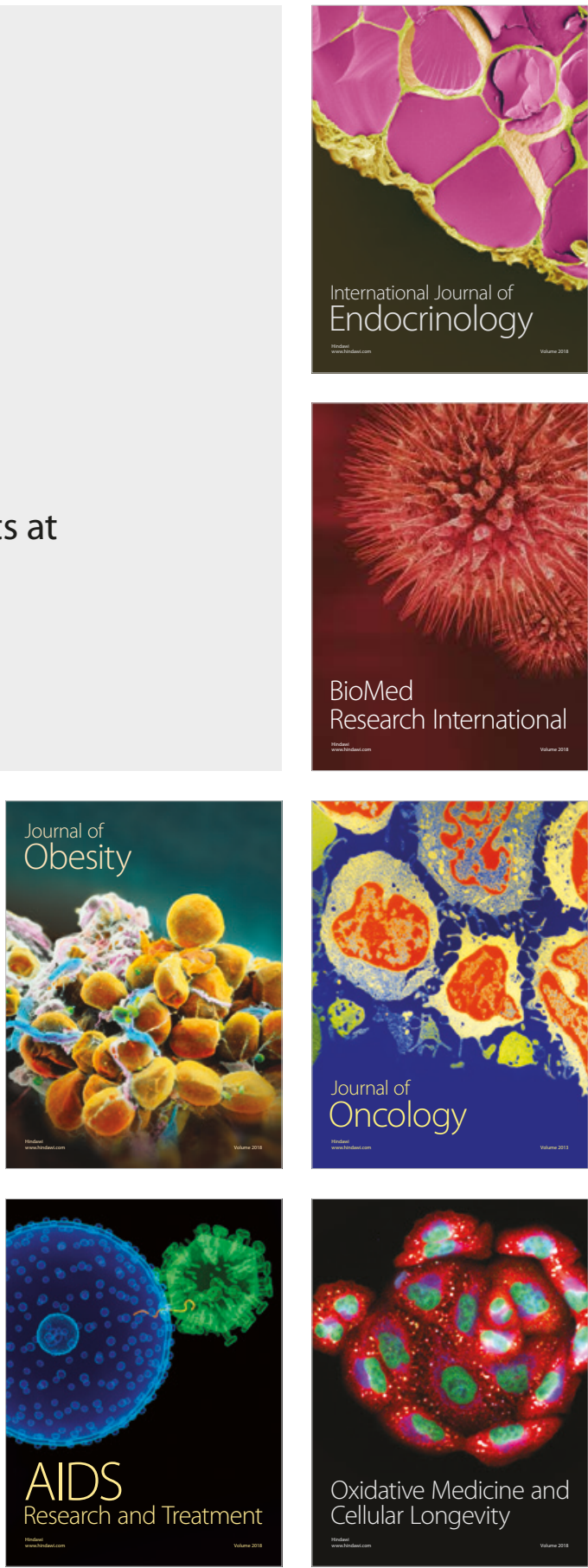